\title{
SMALL-ENERGY ANALYSIS FOR THE SELFADJOINT MATRIX SCHRÖDINGER OPERATOR ON THE HALF LINE
}

\author{
Tuncay Aktosun \\ Department of Mathematics \\ University of Texas at Arlington \\ Arlington, TX 76019-0408, USA \\ aktosun@uta.edu \\ Martin Klaus \\ Department of Mathematics \\ Virginia Tech \\ Blacksburg, VA 24061, USA \\ mklaus@math.vt.edu \\ Ricardo Weder $^{\dagger}$ \\ Instituto de Investigaciones en Matemáticas Aplicadas y en Sistemas \\ Universidad Nacional Autónoma de México \\ Apartado Postal 20-726, IIMAS-UNAM, México DF 01000, México \\ weder@unam.mx
}

\begin{abstract}
The matrix Schrödinger equation with a selfadjoint matrix potential is considered on the half line with the most general selfadjoint boundary condition at the origin. When the matrix potential is integrable and has a first moment, it is shown that the corresponding scattering matrix is continuous at zero energy. An explicit formula is provided for the scattering matrix at zero energy. The small-energy asymptotics are established also for the related Jost matrix, its inverse, and various other quantities relevant to the corresponding direct and inverse scattering problems.
\end{abstract}

\section{Mathematics Subject Classification (2010): 34L25 34L40 81U05 81Uxx}

Keywords: matrix Schrödinger equation on the half line, selfadjoint boundary condition, Jost matrix, small-energy limit, scattering matrix, Jost solution, quantum wires, quantum graphs

Short title: Half-line matrix Schrödinger equation

\footnotetext{
$\dagger$ Fellow Sistema Nacional de Investigadores
} 


\section{INTRODUCTION}

Consider the matrix Schrödinger equation on the half line

$$
-\psi^{\prime \prime}+V(x) \psi=k^{2} \psi, \quad x \in(0,+\infty),
$$

where the prime denotes the derivative with respect to the spatial coordinate $x$ and the potential $V$ is a $n \times n$ matrix-valued function belonging to class $L_{1}^{1}\left(\mathbf{R}^{+}\right)$with $\mathbf{R}^{+}:=(0,+\infty)$. Note that $V \in L_{j}^{1}(\mathbf{I})$ means that each entry of the matrix $V$ is Lebesgue measurable on the interval I and

$$
\int_{\mathbf{I}} d x(1+|x|)^{j}|| V(x)||<+\infty,
$$

where $\|V(x)\|$ denotes a matrix norm. Clearly, a matrix-valued function belongs to $L_{1}^{1}\left(\mathbf{R}^{+}\right)$ if and only if each entry of that matrix belongs to $L_{1}^{1}\left(\mathbf{R}^{+}\right)$. Note that $V$ is not assumed to be real valued, but we impose the condition that it is selfadjoint, i.e.

$$
V=V^{\dagger}
$$

where the dagger denotes the adjoint (complex conjugate and matrix transpose). Without loss of any generality we can view the wavefunction $\psi(k, x)$ appearing in (1.1) either as a vector-valued function with $n$ components or as an $n \times p$ matrix-valued function for some $p$ with $1 \leq p \leq n$.

We are interested in studying (1.1) with a selfadjoint potential $V$ in $L_{1}^{1}\left(\mathbf{R}^{+}\right)$under the most general selfadjoint boundary condition at $x=0$. This is the generalization of the scalar version (with $n=1$ ) of the corresponding problem, where the most general selfadjoint boundary condition at $x=0$ can be stated as $[5,8,16,30]$

$$
(\cos \theta) \psi(0)+(\sin \theta) \psi^{\prime}(0)=0,
$$

where the parameter $\theta$ takes values in the interval $(0, \pi]$. The special choice $\theta=\pi$ corresponds to the Dirichlet boundary condition, and the choice $\theta=\pi / 2$ corresponds to the Neumann boundary condition. 
A formulation of the most general selfadjoint boundary condition at $x=0$ for $(1.1)$ was stated in $[23,24]$ as

$$
A_{1} \psi(0)+B_{1} \psi^{\prime}(0)=0
$$

such that the constant $n \times n$ matrices $A_{1}$ and $B_{1}$ satisfy

$$
\begin{gathered}
A_{1} B_{1}^{\dagger}=B_{1} A_{1}^{\dagger}, \\
\operatorname{rank}\left[A_{1} \quad B_{1}\right]=n,
\end{gathered}
$$

i.e. $A_{1} B_{1}^{\dagger}$ is selfadjoint and the $n \times 2 n$ matrix $\left[\begin{array}{ll}A_{1} & B_{1}\end{array}\right]$ has rank $n$.

Another formulation of the most general selfadjoint boundary condition at $x=0$ for (1.1) was stated in [18-20] in terms of a constant $n \times n$ unitary matrix $U_{2}$ as

$$
-B_{2}^{\dagger} \psi(0)+A_{2}^{\dagger} \psi^{\prime}(0)=0
$$

where the auxiliary constant $n \times n$ matrices $A_{2}$ and $B_{2}$ are given by

$$
A_{2}:=\frac{1}{2}\left(U_{2}+I_{n}\right), \quad B_{2}:=\frac{i}{2}\left(U_{2}-I_{n}\right),
$$

with $I_{n}$ denoting the $n \times n$ identity matrix. It can directly be verified that the matrices $A_{2}$ and $B_{2}$ satisfy

$$
\begin{gathered}
A_{2}^{\dagger} A_{2}=A_{2} A_{2}^{\dagger}, \quad B_{2}^{\dagger} B_{2}=B_{2} B_{2}^{\dagger}, \quad A_{2} B_{2}^{\dagger}=B_{2} A_{2}^{\dagger}, \quad A_{2}^{\dagger} B_{2}=B_{2}^{\dagger} A_{2}, \\
A_{2} A_{2}^{\dagger}+B_{2} B_{2}^{\dagger}=I_{n}, \quad A_{2}+i B_{2}=I_{n}, \quad A_{2}-i B_{2}=U_{2} .
\end{gathered}
$$

We ourselves find it convenient to state the most general selfadjoint boundary condition at $x=0$ for (1.1) in terms of constant $n \times n$ matrices $A_{3}$ and $B_{3}$ such that

$$
\begin{gathered}
-B_{3}^{\dagger} \psi(0)+A_{3}^{\dagger} \psi^{\prime}(0)=0, \\
-B_{3}^{\dagger} A_{3}+A_{3}^{\dagger} B_{3}=0, \\
A_{3}^{\dagger} A_{3}+B_{3}^{\dagger} B_{3}>0,
\end{gathered}
$$


i.e. $A_{3}^{\dagger} B_{3}$ is selfadjoint and the selfadjoint matrix $\left(A_{3}^{\dagger} A_{3}+B_{3}^{\dagger} B_{3}\right)$ is positive. Note that (1.13) implies the existence of a unique positive matrix $E_{3}$ defined as

$$
E_{3}:=\left(A_{3}^{\dagger} A_{3}+B_{3}^{\dagger} B_{3}\right)^{1 / 2}
$$

such that $E_{3}$ is selfadjoint and invertible, and hence

$$
E_{3}=E_{3}^{\dagger}, \quad\left(E_{3}^{\dagger}\right)^{-1}\left(A_{3}^{\dagger} A_{3}+B_{3}^{\dagger} B_{3}\right) E_{3}^{-1}=I_{n}
$$

Let us define the matrices $C_{3}$ and $H_{3}$ as follows

$$
C_{3}:=\left[\begin{array}{cc}
B_{3} & A_{3} \\
A_{3} & -B_{3}
\end{array}\right], \quad H_{3}:=C_{3}\left[\begin{array}{cc}
E_{3}^{-1} & 0 \\
0 & E_{3}^{-1}
\end{array}\right]
$$

With the help of (1.14) and (1.15), it can be checked that $H_{3}^{\dagger} H_{3}=I_{2 n}$ and hence $H_{3}$ is unitary. Thus, we must have $H_{3} H_{3}^{\dagger}=I_{2 n}$, yielding

$$
A_{3} E_{3}^{-2} A_{3}^{\dagger}+B_{3} E_{3}^{-2} B_{3}^{\dagger}=I_{2 n}, \quad B_{3} E_{3}^{-2} A_{3}^{\dagger}-A_{3} E_{3}^{-2} B_{3}^{\dagger}=0 .
$$

We will mainly be working with the formulation given in (1.11)-(1.13) and hence later we will drop the subscripts in $A_{3}, B_{3}, E_{3}$ and simply write $A, B, E$ if there is no confusion.

Let us note that one can multiply the boundary conditions stated in (1.4), (1.7), and (1.11) on the left by an invertible matrix $D$ without changing the most general selfadjoint boundary condition at $x=0$. For example, for (1.11), by dropping the subscript 3 , that left multiplication can be described via the transformation

$$
(A, B) \mapsto(\tilde{A}, \tilde{B}):=\left(A D^{\dagger}, B D^{\dagger}\right)
$$

for which we have

$$
\begin{gathered}
\left(-B^{\dagger} A+A^{\dagger} B\right) \mapsto-D^{-1}\left(-\tilde{B}^{\dagger} \tilde{A}+\tilde{A}^{\dagger} \tilde{B}\right)\left(D^{\dagger}\right)^{-1}, \\
\left(A^{\dagger} A+B^{\dagger} B\right) \mapsto D^{-1}\left(\tilde{A}^{\dagger} \tilde{A}+\tilde{B}^{\dagger} \tilde{B}\right)\left(D^{\dagger}\right)^{-1},
\end{gathered}
$$


and hence (1.11)-(1.13) hold with $(\tilde{A}, \tilde{B})$ appearing instead of $(A, B)$. Thus, the transformed pair $(\tilde{A}, \tilde{B})$ can be used instead of $(A, B)$ in the formulation of the selfadjoint boundary condition.

Our primary goal is to establish, under the most general selfadjoint boundary condition at $x=0$, the small- $k$ asymptotics of various quantities related to (1.1) such as scattering solutions, the Jost matrix, the inverse of the Jost matrix, and the scattering matrix. The small- $k$ analysis for (1.1) has been lacking in the literature even though the relevant results are crucial in the study of the corresponding direct and inverse scattering problems. The direct scattering problem for (1.1) is to determine the scattering matrix and the boundstate information when the matrix potential $V$ and the selfadjoint boundary condition are known. On the other hand, the inverse scattering problem is to recover the potential and the boundary condition from an appropriate set of scattering data. In some sense, our paper can be considered as a complement to the study by Agranovich and Marchenko [1], where the inverse scattering problem is analyzed only under the Dirichlet boundary condition but with attention to the behavior at $k=0$. Our study can also be considered as a complement to the study by Harmer [18-20] where the most general selfadjoint boundary condition (1.7) is used to investigate the inverse problem for (1.1) but the small- $k$ analysis is omitted. We refer the reader to $[2,22]$ for similar small- $k$ analyses for the scalar radial Schrödinger equation, to [3,21] for the scalar full-line Schrödinger equation, to [4] for the matrix full-line Schrödinger equation, and to [5] for the radial Schrödinger equation with the most general selfadjoint boundary condition at the origin.

Let us look at the definition of the Jost matrix $J(k)$ given in (4.3). When $V$ is selfadjoint and belongs to $L_{1}^{1}\left(\mathbf{R}^{+}\right)$, it is already known $[1,4]$ that the right hand side in (4.3) is continuous in $k \in \overline{\mathbf{C}^{+}}$, where we use $\mathbf{C}$ for the complex plane, $\mathbf{C}^{+}$for the upper half complex plane, and $\overline{\mathbf{C}^{+}}:=\mathbf{C}^{+} \cup \mathbf{R}$. Thus, $J(0)$ exists. Let us also look at the definition of the scattering matrix $S(k)$ given in (4.6) in terms of the Jost matrix $J(k)$. In case $J(0)$ is invertible, it is clear from (4.6) that $S(0)=-I_{n}$. However, if $J(0)$ is not invertible, it is unclear whether $S(k)$ is continuous at $k=0$ and what the value of $S(0)$ is in case the 
continuity at $k=0$ is assured. Our paper mainly concentrates on the case when $J(0)^{-1}$ does not exist. We prove that $S(k)$ is indeed continuous at $k=0$ and we determine the value of $S(0)$, which in general is different from $-I_{n}$. In case $J(0)$ is invertible, our results reduce to the easy case with $S(0)=-I_{n}$.

Let us note that $J(0)$ is not invertible if and only if the determinant $\operatorname{det}[J(0)]$ is zero. In the scalar case (i.e. when $n=1$ ) this is the analog of $F_{\theta}(0)=0$, where $F_{\theta}(k)$ is the Jost function appearing in (4.1). The case $F_{\theta}(0)=0$ is known as the "exceptional case," and the case $F_{\theta}(0) \neq 0$ is known as the "generic case." Hence, in our paper we concentrate on the "exceptional case" for (1.1), namely the case when $J(0)$ is not invertible. In the "generic case" it is already known and easy to see that $S(k)$ is continuous at $k=0$ and $S(0)=-I_{n}$. In the exceptional case, by expressing $J(k)$ as in (6.17) in terms of a related matrix $\mathcal{Z}(k)$, and by writing the scattering matrix $S(k)$ as in $(6.19)$ in terms of $\mathcal{Z}(-k)$ and $\mathcal{Z}(k)^{-1}$, we are able to prove the continuity of $S(k)$ at $k=0$ and evaluate $S(0)$.

We remind the reader that the continuity of the scattering matrix in the exceptional case is not an easy matter. For example, in the full-line scalar case, Deift and Trubowitz [9] stated that the characterization of the scattering data given by Faddeev [12] might not hold and in fact even the continuity of the scattering matrix was not clear when the real-valued potential belonged to $L_{1}^{1}(\mathbf{R})$ and they introduced the stronger condition that the potential belonged to $L_{2}^{1}(\mathbf{R})$. The proof of the continuity of the scattering matrix when the potential belongs to $L_{1}^{1}(\mathbf{R})$ was given later. For further details we refer the reader to $[3,21]$ and the references therein.

The matrix Schrödinger equation (1.1) has direct relevance to scattering in quantum mechanics involving particles of internal structures as spins, scattering on graphs $[6,7,11,14,15,17,25-28]$, and quantum wires [23,24]. For example, the problem under study describes $n$ connected very thin quantum wires forming a one-vertex graph with open ends. A linear boundary condition is imposed at the vertex and the behavior on each wire is governed by the Schrödinger operator. The problem has physical relevance to designing 
elementary gates in quantum computing and nanotubes for microscopic electronic devices, where, for example, strings of atoms may form a star-shaped graph. For the details we refer the reader to $[23,24]$ and the references therein.

Our paper is organized as follows. In Section 2 we show that the three selfadjoint boundary condition formulations given in Section 1 are equivalent. In Section 3 we introduce various $n \times n$ matrix solutions to (1.1) and state their properties relevant to the small- $k$ analysis of (1.1). In Section 4 we introduce the Jost matrix $J(k)$ and the scattering matrix $S(k)$. In Section 5 we obtain various results that are crucial in determining the small- $k$ asymptotics of the Jost matrix, its inverse, and the scattering matrix. In Section 6 we provide the small- $k$ asymptotics for $J(k), J(k)^{-1}$, and $S(k)$, and we prove that $S(k)$ is continuous at $k=0$. Finally, in Section 7 we provide some examples to illustrate the theory presented.

\section{EQUIVALENCE OF BOUNDARY CONDITION FORMULATIONS}

In Section 1 we have stated the three formulations of the most general selfadjoint boundary conditions at $x=0$ for $(1.1)$ :

(a) The formulation (1.4)-(1.6) stated in [23,24].

(b) The formulation (1.7) and (1.8) stated in [18-20].

(c) Our own formulation stated as (1.11)-(1.13).

In this section we show that those three formulations are equivalent.

Theorem 2.1 The three formulations (a), (b), and (c) of the most general selfadjoint boundary conditions at $x=0$ for (1.1) are all equivalent.

PROOF: With the help of (1.17), we can relate (1.11)-(1.14) to (1.7) and (1.8) by letting

$$
U_{2}=\left(A_{3}-i B_{3}\right) E_{3}^{-2}\left(A_{3}^{\dagger}-i B_{3}^{\dagger}\right)
$$

and we can verify (1.7) and (1.8) with the help of (1.15) and (1.16). Hence, we have shown that (c) implies (b). Next, we will show that (b) implies (a). Let $A_{1}=-B_{2}^{\dagger}$ and $B_{1}=A_{2}^{\dagger}$. 
Then (1.7) implies (1.4). Furthermore, the last equality in (1.9) yields (1.5). Let

$$
C_{2}:=\left[\begin{array}{cc}
B_{2} & A_{2} \\
A_{2} & -B_{2}
\end{array}\right] \text {. }
$$

We then get

$$
C_{2}^{\dagger} C_{2}=\left[\begin{array}{cc}
B_{2}^{\dagger} B_{2}+A_{2}^{\dagger} A_{2} & B_{2}^{\dagger} A_{2}-A_{2}^{\dagger} B_{2} \\
A_{2}^{\dagger} B_{2}-B_{2}^{\dagger} A_{2} & A_{2}^{\dagger} A_{2}+B_{2}^{\dagger} B_{2}
\end{array}\right]=\left[\begin{array}{cc}
I_{n} & 0 \\
0 & I_{n}
\end{array}\right],
$$

where we have used (1.9) and (1.10). Thus, $C_{2}$ is unitary and has rank $2 n$. As a result the block matrix $\left[\begin{array}{ll}B_{2}^{\dagger} & A_{2}^{\dagger}\end{array}\right]$ has rank $n$. That matrix is in fact equal to $\left[\begin{array}{ll}-A_{1} & B_{1}\end{array}\right]$, and changing the signs in the first $n$ columns does not affect its rank. Thus, $\left[\begin{array}{ll}A_{1} & B_{1}\end{array}\right]$ has rank $n$ and (1.6) is satisfied. Hence, we have shown that (b) implies (a). Finally, let us show that (a) implies (c). Let $B_{3}=-A_{1}^{\dagger}$ and $A_{3}=B_{1}^{\dagger}$. Then, (1.4) implies (1.11), and (1.5) yields (1.12). Note that

$$
A_{3}^{\dagger} A_{3}+B_{3}^{\dagger} B_{3}=\left[\begin{array}{ll}
B_{1} & A_{1}
\end{array}\right]\left[\begin{array}{l}
B_{1}^{\dagger} \\
A_{1}^{\dagger}
\end{array}\right],
$$

and we need to show that the matrix product on the right in (2.1) is positive. This is indeed the case because that matrix product is itself a selfadjoint matrix and zero cannot be one of its eigenvalues. Otherwise, we would have a nonzero eigenvector $v$ with the zero eigenvalue, implying

$$
0=\left\langle v,\left[\begin{array}{ll}
B_{1} & A_{1}
\end{array}\right]\left[\begin{array}{l}
B_{1}^{\dagger} \\
A_{1}^{\dagger}
\end{array}\right] v\right\rangle=\left\langle\left[\begin{array}{c}
B_{1}^{\dagger} \\
A_{1}^{\dagger}
\end{array}\right] v,\left[\begin{array}{l}
B_{1}^{\dagger} \\
A_{1}^{\dagger}
\end{array}\right] v\right\rangle,
$$

with $\langle\cdot, \cdot\rangle$ denoting the standard scalar product. However, (2.2) would then imply that $\left[\begin{array}{l}B_{1}^{\dagger} \\ A_{1}^{\dagger}\end{array}\right] v=0$ and hence the kernel of the matrix $\left[\begin{array}{l}B_{1}^{\dagger} \\ A_{1}^{\dagger}\end{array}\right]$ would contain the nonzero vector $v$. Consequently, the nullity of $\left[\begin{array}{l}B_{1}^{\dagger} \\ A_{1}^{\dagger}\end{array}\right]$ would be at least 1 . Since the nullity and the rank must add up to $n$, the rank would have to be strictly less than $n$, violating the fact that the rank of that matrix is exactly $n$ because of (1.6). Thus, (a) implies (c).

In the following proposition we state a fourth equivalent formulation of the most general selfadjoint boundary condition at $x=0$ for (1.1). 
Proposition 2.2 The three formulations (a), (b), and (c) of the most general selfadjoint boundary condition are also equivalent to the formulation in terms of two constant $n \times n$ matrices $A_{4}$ and $B_{4}$ as

$$
-B_{4}^{\dagger} \psi(0)+A_{4}^{\dagger} \psi^{\prime}(0)=0,
$$

such that the matrix $C_{4}$ is unitary, where we have defined

$$
C_{4}:=\left[\begin{array}{cc}
B_{4} & A_{4} \\
A_{4} & -B_{4}
\end{array}\right] .
$$

PROOF: Because of Theorem 2.1, it is sufficient to prove the equivalence of (2.3)-(2.4) with (1.11)-(1.13). Suppose that (2.3)-(2.4) hold. Then, letting $A_{3}=A_{4}$ and $B_{3}=B_{4}$, we get (1.11) and we obtain (1.12) and (1.13) from the unitarity of $C_{4}$. Conversely, suppose (1.11)-(1.13) hold. By letting $A_{4}=A_{3} E_{3}^{-1}$ and $B_{4}=B_{3} E_{3}^{-1}$, we observe that (1.12) and (1.15) yield $C_{4}^{\dagger} C_{4}=I_{2 n}$, implying the unitarity of $C_{4}$.

We have seen in (1.7) and (1.8) that the most general selfadjoint boundary condition for (1.1) can be stated in terms of a unitary matrix. There are certainly other choices for such a unitary matrix besides $U_{2}$ appearing in (1.8). For example, in terms of a unitary matrix $U_{5}$, instead of (1.7) and (1.8) we can use

$$
-B_{5}^{\dagger} \psi(0)+A_{5}^{\dagger} \psi^{\prime}(0)=0,
$$

where the auxiliary constant $n \times n$ matrices $A_{5}$ and $B_{5}$ are given by

$$
A_{5}:=\frac{i}{2}\left(U_{5}-U_{5}^{\dagger}\right), \quad B_{5}:=\frac{1}{2}\left(U_{5}+U_{5}^{\dagger}\right) .
$$

As seen from (2.6) we can simultaneously diagonalize $U_{5}$ into the form

$$
U_{5}=\operatorname{diag}\left\{e^{i \theta_{1}}, e^{i \theta_{2}}, \ldots, e^{i \theta_{n}}\right\}
$$

for some real-valued parameters $\theta_{j}$. Then, the boundary condition given in (2.5) is separated into the $n$ conditions given by

$$
\left(\cos \theta_{j}\right) \psi_{j}(0)+\left(\sin \theta_{j}\right) \psi_{j}^{\prime}(0)=0, \quad j=1, \ldots, n,
$$


where $\psi_{j}$ denotes the $j$ th column of the $n \times n$ matrix solution $\psi$. Similarly, for the choice (1.8) for $\left(A_{2}, B_{2}\right)$ in terms of a unitary matrix $U_{2}$, by diagonalizing $U_{2}$ as in (2.7), we can express (1.7) as $n$ separate boundary conditions given by

$$
\left[\sin \left(\theta_{j} / 2\right)\right] \psi_{j}(0)+\left[\cos \left(\theta_{j} / 2\right)\right] \psi_{j}^{\prime}(0)=0, \quad j=1, \ldots, n
$$

\section{PRELIMINARIES}

In this section we introduce certain $n \times n$ matrix solutions to (1.1) and state their properties that will be useful later on. We state the results without proofs and refer the reader to the appropriate references such as [1,4] for details. Let us recall that we use the boundary conditions stated in (1.11)-(1.13) without the subscript 3 . When $V$ is selfadjoint and belongs to $L_{1}^{1}\left(\mathbf{R}^{+}\right)$, the matrix Schrödinger equation (1.1) has various $n \times n$ matrix solutions satisfying certain initial conditions or certain asymptotic conditions, and the existence of such solutions are already known.

The Jost solution to (1.1) is the $n \times n$ matrix solution satisfying, for $k \in \overline{\mathbf{C}^{+}} \backslash\{0\}$, the asymptotics

$$
f(k, x)=e^{i k x}\left[I_{n}+o(1)\right], \quad f^{\prime}(k, x)=i k e^{i k x}\left[I_{n}+o(1)\right], \quad x \rightarrow+\infty .
$$

It satisfies the integral equation

$$
f(k, x)=e^{i k x} I_{n}+\frac{1}{k} \int_{x}^{\infty} d y \sin k(y-x) V(y) f(k, y),
$$

and it is known $[1,4]$ that $f(k, x)$ and $f^{\prime}(k, x)$ are analytic in $k \in \mathbf{C}^{+}$and continuous in $k \in \overline{\mathbf{C}^{+}}$for each fixed $x$. The zero-energy Jost solution $f(0, x)$ satisfies

$$
f(0, x)=I_{n}+\int_{x}^{\infty} d y(y-x) V(y) f(0, y)
$$

and it is known $[1,4]$ that $f(0, x)$ is a bounded solution to the $n \times n$ matrix-valued zeroenergy Schrödinger equation

$$
-\psi^{\prime \prime}+V(x) \psi=0, \quad x \in(0,+\infty)
$$


satisfying

$$
f(0, x)=I_{n}+o(1), \quad f^{\prime}(0, x)=o(1 / x), \quad x \rightarrow+\infty .
$$

It is also known [1,4] that (3.2) has an $n \times n$ matrix solution $g(0, x)$ satisfying

$$
g(0, x)=x\left[I_{n}+o(1)\right], \quad g^{\prime}(0, x)=I_{n}+o(1), \quad x \rightarrow+\infty .
$$

Thus, the $2 n$ columns of $f(0, x)$ and $g(0, x)$ form a fundamental set of solutions to (3.2), and any vector solution $\phi(x)$ to (3.2) can be expressed as

$$
\phi(x)=f(0, x) \xi+g(0, x) \eta, \quad x \in(0,+\infty)
$$

where the constant vectors $\xi$ and $\eta$ in $\mathbf{C}^{n}$ are uniquely determined by $\phi(x)$. We see from (3.4) and (3.5) that any solution to (3.2) that behaves as $o(x)$ as $x \rightarrow+\infty$ must be a bounded solution.

There are various $n \times n$ matrix solutions to (1.1) defined via specifying some constant initial conditions at a finite $x$-value. As a result, such solutions are analytic in $k$ in the entire complex plane for each fixed $x$. Because of their analyticity such solutions are usually called "regular" solutions. The $n \times n$ regular solution $\varphi(k, x)$ satisfies the initial conditions

$$
\varphi(k, 0)=A, \quad \varphi^{\prime}(k, 0)=B
$$

where $A$ and $B$ are the matrices appearing in (1.11). It satisfies the integral relation

$$
\varphi(k, x)=A \cos k x+B \frac{\sin k x}{k}+\frac{1}{k} \int_{0}^{x} d y \sin k(x-y) V(y) \varphi(k, y) .
$$

Let us define two additional regular $n \times n$ matrix-valued solutions to (1.1), namely $C(k, x)$ and $S(k, x)$ with initial conditions at $x=a$, at which the matrix $f(0, a)$ is invertible. The existence of such an $a$-value is assured by the fact that $f(0, x)=I_{n}+o(1)$ as $x \rightarrow+\infty$ and hence $f(0, x)$ is invertible at least for large $x$-values. In fact, if $f(0, a)^{-1}$ exists, then we must have the existence of $f(k, a)^{-1}$ in the vicinity of $k=0$ in $\overline{\mathbf{C}^{+}}$. This is because for each fixed $x$-value it is known [4] that $f(k, x)$ is a continuous function of $k \in \overline{\mathbf{C}^{+}}$. Hence, 
$\operatorname{det}[f(k, a)]$ is a continuous function of $k$ and if it is nonzero at $k=0$ it must be nonzero in the vicinity of $k=0$. Thus, we conclude that

$$
f(k, a)=f(0, a)+o(1), \quad f(k, a)^{-1}=f(0, a)^{-1}+o(1), \quad k \rightarrow 0 \text { in } \overline{\mathbf{C}^{+}} .
$$

The cosine-like solution $C(k, x)$ satisfies the initial conditions

$$
C(k, a)=I_{n}, \quad C^{\prime}(k, a)=0,
$$

and the sine-like solution $S(k, x)$ satisfies

$$
S(k, a)=0, \quad S^{\prime}(k, a)=I_{n}
$$

Thus, we have the integral representations

$$
\begin{aligned}
& C(k, x)=I_{n} \cos k(x-a)+\frac{1}{k} \int_{a}^{x} d y \sin k(x-y) V(y) C(k, y), \\
& S(k, x)=I_{n} \frac{\sin k(x-a)}{k}+\frac{1}{k} \int_{a}^{x} d y \sin k(x-y) V(y) S(k, y) .
\end{aligned}
$$

Note that we suppress the dependence on $a$ in our notation for such solutions.

We define another $n \times n$ regular solution to $(1.1), \omega(k, x)$, which satisfies the initial conditions

$$
\omega(k, a)=f(0, a), \quad \omega^{\prime}(k, a)=f^{\prime}(0, a) .
$$

Again we suppress the dependence on $a$ in our notation for $\omega(k, x)$. Note that

$$
\omega(0, x)=f(0, x), \quad x \in \mathbf{R}^{+},
$$

because both sides satisfy (1.1) when $k=0$ and they both satisfy the same initial conditions at $x=a$ given in (3.13). It is seen from (3.9), (3.10), and (3.13) that

$$
\omega(k, x)=C(k, x) f(0, a)+S(k, x) f^{\prime}(0, a)
$$

where $f(k, x)$ is the Jost solution appearing in $(3.1)$. 
Let us note that our regular solutions satisfy for $k \in \mathbf{C}$

$$
\varphi(-k, x)=\varphi(k, x), \quad C(-k, x)=C(k, x), \quad S(-k, x)=S(k, x), \quad \omega(-k, x)=\omega(k, x) .
$$

This is because $k$ appears as $k^{2}$ in (1.1) and the initial values of those solutions are independent of $k$, as seen from (3.6), (3.9), (3.10), and (3.13).

Associated with (1.1) we have the adjoint equation

$$
-\psi^{\dagger \prime \prime}+\psi^{\dagger} V(x)=\left(k^{*}\right)^{2} \psi^{\dagger}, \quad x \in(0,+\infty)
$$

where we have used (1.2) and an asterisk denotes complex conjugation. Note that if $\psi(k, x)$ is any solution to (1.1), then $\psi\left( \pm k^{*}, x\right)^{\dagger}$ is a solution to (3.17). Let us also add that if $\psi(k, x)$ has an analytic extension from $k \in \mathbf{R}$ to $k \in \mathbf{C}^{+}$, then $\psi(-k, x)^{\dagger}$ has also an analytic extension from $k \in \mathbf{R}$ to $k \in \mathbf{C}^{+}$, and in fact that extension becomes equal to $\psi\left(-k^{*}, x\right)^{\dagger}$ for $k \in \mathbf{C}^{+}$. A consequence of this is the following. Since it is already known that $f(k, x)$ and $f^{\prime}(k, x)$ are analytic in $k \in \mathbf{C}^{+}, f(-k, x)^{\dagger}$ and $f^{\prime}(-k, x)^{\dagger}$ have analytic extensions from $k \in \mathbf{R}$ to $k \in \mathbf{C}^{+}$given by $f\left(-k^{*}, x\right)^{\dagger}$ and $f^{\prime}\left(-k^{*}, x\right)^{\dagger}$, respectively.

Let $[F ; G]:=F G^{\prime}-F^{\prime} G$ denote the Wronskian. It can directly be verified that for any $n \times p$ solution $\psi(k, x)$ and any $n \times q$ solution $\phi(k, x)$ to $(1.1)$, the Wronskians $\left[\phi\left(k^{*}, x\right)^{\dagger} ; \psi(k, x)\right]$ and $\left[\phi\left(-k^{*}, x\right)^{\dagger} ; \psi(k, x)\right]$ are both independent of $x$. By evaluating the values of the Wronskians at $x=0$ and $x=+\infty$, we can obtain various useful identities. For example, we have

$$
\begin{gathered}
{\left[f( \pm k, x)^{\dagger} ; f( \pm k, x)\right]= \pm 2 i k I_{n}, \quad k \in \mathbf{R},} \\
{\left[f\left(-k^{*}, x\right)^{\dagger} ; f(k, x)\right]=0, \quad k \in \overline{\mathbf{C}^{+}} .}
\end{gathered}
$$

\section{THE JOST MATRIX AND THE SCATTERING MATRIX}

In this section we introduce the Jost matrix and the scattering matrix for (1.1) with a selfadjoint matrix potential $V$ in $L_{1}^{1}\left(\mathbf{R}^{+}\right)$and with the selfadjoint boundary condition 
(1.11)-(1.13). We also present certain preliminary results needed later on to analyze the small- $k$ limits of these two matrices and of the inverse of the Jost matrix.

Recall that the Jost function $F_{\theta}$ corresponding to (1.3) in the scalar case, i.e. when $n=1$ in $(1.1)$, is defined with the help of the Jost solution $f(k, x)$ as $[5,16,29,31]$

$$
F_{\theta}(k):=\left\{\begin{array}{l}
-i\left[f^{\prime}(k, 0)+(\cot \theta) f(k, 0)\right], \quad \theta \in(0, \pi), \\
f(k, 0), \quad \theta=\pi .
\end{array}\right.
$$

We will define the matrix analog of the Jost function, which is called the Jost matrix, so that it reduces to the familiar Jost function when $n=1$. Recall also that the scattering matrix in the scalar case is defined as $[5,16,29,31]$

$$
S_{\theta}(k):= \begin{cases}-\frac{F_{\theta}(-k)}{F_{\theta}(k)}, & \theta \in(0, \pi), \\ \frac{F_{\theta}(-k)}{F_{\theta}(k)}, & \theta=\pi .\end{cases}
$$

The reason behind the sign difference in (4.2) in the Dirichlet case (i.e. when $\theta=\pi$ ) is that (4.2) ensures that $S(k) \rightarrow 1$ as $V \rightarrow 0$, which is a consequence of the fact that the perturbed and unperturbed Hamiltonians satisfy the same selfadjoint boundary condition at $x=0$. We will define the scattering matrix by generalizing (4.2) to the matrix case. For simplicity, we will suppress the dependence of the Jost matrix and the scattering matrix on the boundary-condition parametrization $(A, B)$, and we will use the notation $J(k)$ for the Jost matrix instead of $J_{(A, B)}(k)$ and also write $S(k)$ for the scattering matrix instead of $S_{(A, B)}(k)$. Note that we earlier used $S(k, x)$ in $(3.10)$ to denote the sine-like regular solution to (1.1), which should not be confused with the notation $S(k)$ used for the scattering matrix.

Define the Jost matrix $J(k)$ for $k \in \overline{\mathbf{C}^{+}}$as

$$
J(k):=\left[f\left(-k^{*}, x\right)^{\dagger} ; \varphi(k, x)\right]=f\left(-k^{*}, 0\right)^{\dagger} B-f^{\prime}\left(-k^{*}, 0\right)^{\dagger} A,
$$

where $f(k, x)$ is the Jost solution appearing in $(3.1), \varphi(k, x)$ is the regular solution appearing in (3.6), and $A$ and $B$ are the matrices appearing in (1.11)-(1.13) and (3.6). Note that 
$J$ is not uniquely determined by the potential $V$ and the selfadjoint boundary condition (1.11)-(1.13). This is because (1.11)-(1.13) are invariant under the transformation (1.17), and hence we have $J \mapsto J D^{\dagger}$ under (1.17) indicating that the definition for $J$ in (4.3) is unique up to a right multiplication by a constant invertible matrix. On the other hand, such a postmultiplication does not change the zeros in $\mathbf{C}^{+}$of the determinant of $J(k)$. Those zeros correspond $[1,19]$ to the bound-state energies of $(1.1)$ with the boundary condition (1.11)-(1.13), and hence the bound-state energies are still uniquely determined by $(4.3)$.

Theorem 4.1 If $V$ is selfadjoint and belongs to $L_{1}^{1}\left(\mathbf{R}^{+}\right)$, then the Jost matrix $J(k)$ is invertible for $k \in \mathbf{R} \backslash\{0\}$.

PROOF: Even though a proof is available [19], for the benefit of the reader we outline a proof of our own. For $k \in \mathbf{R}$ define

$$
L(k):=f^{\prime}(-k, 0)^{\dagger} B E^{-2}+f(-k, 0)^{\dagger} A E^{-2},
$$

where $E$ is the matrix $E_{3}$ appearing in (1.14). With the help of (1.16), (3.18), (4.3), and (4.4) one can show that

$$
J(k) L(k)^{\dagger}-L(k) J(k)^{\dagger}=\left.\left[f(-k, x)^{\dagger} ; f(-k, x)\right]\right|_{x=0}=-2 i k I_{n}, \quad k \in \mathbf{R} .
$$

If $J(k)$ were noninvertible at some real nonzero $k_{0}$, then the rows of $J\left(k_{0}\right)$ would be linearly dependent and hence we would have $u^{\dagger} J\left(k_{0}\right)=0$ for some nonzero vector $u \in \mathbf{C}^{n}$ as well as $J\left(k_{0}\right)^{\dagger} u=0$. However, because of (4.5) this would imply

$$
0=u^{\dagger} J\left(k_{0}\right) L\left(k_{0}\right)^{\dagger} u-u^{\dagger} L\left(k_{0}\right) J\left(k_{0}\right)^{\dagger} u=-2 i k_{0} u^{\dagger} u=-2 i k_{0}\|u\|^{2} \neq 0
$$

which is a contradiction. Thus, $J\left(k_{0}\right)$ must be invertible.

The scattering matrix $S(k)$ is defined as [18-20]

$$
S(k):=-J(-k) J(k)^{-1}, \quad k \in \mathbf{R} \backslash\{0\},
$$


and it is uniquely determined by the boundary condition and the potential $V$. Even though $J(k)$ is uniquely defined only up to a right multiplication by a constant invertible matrix, the unique determination of $S(k)$ is assured because $S(k)$ remains invariant under the transformation (1.17). Note that the domain of $J(k)$ is $k \in \overline{\mathbf{C}^{+}}$because $f(-k, 0)^{\dagger}$ and $f^{\prime}(-k, 0)^{\dagger}$ have analytic extensions from $k \in \mathbf{R}$ to $k \in \mathbf{C}^{+}$and the values of those extensions are $f\left(-k^{*}, 0\right)^{\dagger}$ and $f^{\prime}\left(-k^{*}, 0\right)^{\dagger}$, respectively. On the other hand, in general $S(k)$ is defined only for real $k$ because $J(-k)$ in general cannot be extended from $k \in \mathbf{R}$ to $k \in \mathbf{C}^{+}$. Furthermore, the existence of $S(k)$ when $k=0$ needs to be studied separately because, as we have seen in Theorem 4.1, the existence of $J(k)^{-1}$ is assured only for $k \in \mathbf{R} \backslash\{0\}$ and it cannot easily be inferred from (4.6) whether $S(k)$ has a limit as $k \rightarrow 0$ when $J(0)^{-1}$ does not exist.

In order to understand the small- $k$ behavior of $J(k)$ and $S(k)$, it is instructive to analyze first the case when the potential $V$ is identically zero in (1.1). In that case, we have $f(k, x)=e^{i k x} I_{n}$, and hence (4.3) and (4.6) yield

$$
J(k)=B-i k A, \quad[J(k)]^{-1}=(B-i k A)^{-1}, \quad S(k)=-(B+i k A)(B-i k A)^{-1} .
$$

Let us use the representation (2.6) for $(A, B)$ with the diagonal form of $U$ given in (2.7). We then obtain

$$
\begin{gathered}
A=-\operatorname{diag}\left\{\sin \theta_{1}, \ldots, \sin \theta_{n}\right\}, \quad B=\operatorname{diag}\left\{\cos \theta_{1}, \ldots, \cos \theta_{n}\right\}, \\
J(k)=\operatorname{diag}\left\{J_{1}(k), \ldots, J_{n}(k)\right\}, \quad S(k)=\operatorname{diag}\left\{S_{1}(k), \ldots, S_{n}(k)\right\},
\end{gathered}
$$

where we have defined

$$
J_{j}(k):=\cos \theta_{j}+i k \sin \theta_{j}, \quad S_{j}(k):=\frac{-\cos \theta_{j}+i k \sin \theta_{j}}{\cos \theta_{j}+i k \sin \theta_{j}} .
$$

As seen from (4.7), in the Dirichlet case (i.e. when $\theta_{j}=\pi$ ) we have

$$
J_{j}(k)=-1, \quad\left[J_{j}(k)\right]^{-1}=-1, \quad S_{j}(k)=-1 .
$$


On the other hand, in the Neumann case (i.e. when $\theta_{j}=\pi / 2$ ) we have

$$
J_{j}(k)=i k, \quad\left[J_{j}(k)\right]^{-1}=\frac{1}{i k}, \quad S_{j}(k)=1 .
$$

Note that, in the Neumann case, $J_{j}(0)$ vanishes linearly as $k \rightarrow 0$ and it is not an invertible matrix; however, $S_{j}(0)$ is still well defined because $J(-k)[J(k)]^{-1}$ has a well-defined limit as $k \rightarrow 0$. It is somehow disturbing that in the Dirichlet case, $S_{j}(0) \neq 1$ and in fact $S_{j}(0)=-1$, which is exactly the opposite of the scalar case as seen from (4.2). The explanation for the discrepancy is that the unperturbed Hamiltonian in the matrix case is chosen to satisfy the Neumann boundary condition, which is compatible with the timedependent derivation of the scattering matrix and motivated by applications in quantum wires; for further elaboration on this point we refer the reader to p. 1566 of [24].

\section{SMALL- $k$ BEHAVIOR}

In preparation for the analysis of the small-energy behavior of the Jost matrix $J(k)$,

its inverse $J(k)^{-1}$, and the scattering matrix $S(k)$, in this section we establish the small- $k$ asymptotics of various quantities related to the regular solutions to (1.1).

We are interested in analyzing the Jost matrix $J(k)$ as $k \rightarrow 0$ in $\overline{\mathbf{C}^{+}}$. From (4.3) we see that

$$
J(0)=f(0,0)^{\dagger} B-f^{\prime}(0,0)^{\dagger} A
$$

and we would like to determine how fast $J(k)$ approaches $J(0)$ and whether $J(k)^{-1}$ exists at $k=0$ and determine its behavior as $k \rightarrow 0$ from $\overline{\mathbf{C}^{+}}$. We would like to know about such small- $k$ behaviors when $V$ is selfadjoint and belongs to $L_{1}^{1}\left(\mathbf{R}^{+}\right)$.

As stated before (3.8), $f(k, a)$ is invertible in the vicinity of $k=0$ in $\overline{\mathbf{C}^{+}}$for some $a$ value. In (4.3) we have defined the Jost matrix in terms of a Wronskian whose value is independent of $x$. As we see below we can write $J(k)^{\dagger}$ in terms of Wronskians evaluated at $x=a$ and involving the solutions $f(k, x), \varphi(k, x)$, and $\omega(k, x)$ appearing in (3.1), (3.6), and (3.13), respectively. 
The following result will be needed later on. By a generic constant, we mean a constant that does not necessarily have the same value in different appearances.

Proposition 5.1 If $V$ is selfadjoint and belongs to $L_{1}^{1}\left(\mathbf{R}^{+}\right)$, then the regular solution $\omega(k, x)$ to (1.1) appearing in (3.13) satisfies

$$
\|\omega(k, x)-\omega(0, x)\| \leq c\left(\frac{|k|(x-a)}{1+|k|(x-a)}\right)^{2} e^{(\operatorname{Im}[k])(x-a)}, \quad k \in \overline{\mathbf{C}^{+}}, \quad x \geq a,
$$

where $c$ is a generic constant.

PROOF: From (3.11), (3.12), and (3.15) we have

$$
\omega(k, x)=f(0, a) \cos k(x-a)+f^{\prime}(0, a) \frac{\sin k(x-a)}{k}+\frac{1}{k} \int_{a}^{x} d y \sin k(x-y) V(y) \omega(k, y) .
$$

Note that (5.3) yields

$$
\omega(0, x)=f(0, a)+(x-a) f^{\prime}(0, a)+\int_{a}^{x} d y(x-y) V(y) \omega(0, y)
$$

and $\omega(0, x)=f(0, x)$ by (3.14). Thus, from (5.4) and its $x$-derivative, with the help of $f^{\prime}(0, x)=o(1 / x)$ as $x \rightarrow+\infty$, we obtain

$$
\begin{gathered}
\int_{a}^{\infty} d y V(y) \omega(0, y)=-f^{\prime}(0, a), \\
\int_{a}^{\infty} d y y V(y) \omega(0, y)=f(0, a)-a f^{\prime}(0, a)-I_{n} .
\end{gathered}
$$

Let us write (5.5) as

$$
f^{\prime}(0, a)=-\int_{a}^{x} d y V(y) \omega(0, y)-\int_{x}^{\infty} d y V(y) \omega(0, y)
$$

Using (5.3), (5.4), and (5.7), we get

$$
\omega(k, x)-\omega(0, x)=K_{1}+K_{2}+K_{3}+K_{4},
$$

where we have defined

$$
K_{1}:=f(0, a)[\cos k(x-a)-1],
$$




$$
\begin{gathered}
K_{2}:=\left[1-\frac{\sin k(x-a)}{k(x-a)}\right](x-a) \int_{x}^{\infty} d y V(y) \omega(0, y) \\
K_{3}:=\frac{1}{k} \int_{a}^{x} d y[\sin k(x-y)-k(x-y)-\sin k(x-a)+k(x-a)] V(y) \omega(0, y), \\
K_{4}:=\frac{1}{k} \int_{a}^{x} d y[\sin k(x-y)] V(y)[\omega(k, y)-\omega(0, y)]
\end{gathered}
$$

For $z \in \overline{\mathbf{C}^{+}}$we have

$$
|\sin z| \leq \frac{c|z| e^{\operatorname{Im}[z]}}{1+|z|}, \quad\left|1-\frac{\sin z}{z}\right| \leq \frac{c|z|^{2} e^{\operatorname{Im}[z]}}{(1+|z|)^{2}}, \quad|1-\cos z| \leq \frac{c|z|^{2} e^{\operatorname{Im}[z]}}{(1+|z|)^{2}}
$$

Using (5.9) and the third estimate of (5.13), we get

$$
\left\|K_{1}\right\| \leq \frac{c|k|^{2}(x-a)^{2} e^{(\operatorname{Im}[k])(x-a)}}{(1+|k|(x-a))^{2}}, \quad k \in \overline{\mathbf{C}^{+}}, \quad x \geq a .
$$

Note that

$$
\begin{aligned}
\left\|(x-a) \int_{x}^{\infty} d y V(y) \omega(0, y)\right\| & \leq\left\|\int_{x}^{\infty} d y(y-a) V(y) \omega(0, y)\right\| \\
& \leq\left\|\int_{x}^{\infty} d y y V(y) \omega(0, y)\right\| .
\end{aligned}
$$

The norms in (5.15) are bounded by a constant due to the facts that $V \in L_{1}^{1}\left(\mathbf{R}^{+}\right)$and $\omega(0, x)$ is bounded as a result of (3.3) and (3.14). Thus, from (5.10), (5.15), and the second estimate in (5.13), we get

$$
\left\|K_{2}\right\| \leq \frac{c|k|^{2}(x-a)^{2} e^{(\operatorname{Im}[k])(x-a)}}{(1+|k|(x-a))^{2}}, \quad k \in \overline{\mathbf{C}^{+}}, \quad x \geq a .
$$

Let us now estimate $K_{3}$ when $k \in \overline{\mathbf{C}^{+}}$and $x \geq a$. We write (5.11) as

$$
K_{3}=\int_{a}^{x} d y \int_{x-y}^{x-a} d z[1-\cos k z] V(y) \omega(0, y)
$$

and use the third estimate of (5.13) and the fact that $x \mapsto x^{2} /(1+x)^{2}$ is an increasing function of $x$ when $x \geq 0$, to obtain

$$
\left\|K_{3}\right\| \leq \frac{c|k|^{2}(x-a)^{2} e^{(\operatorname{Im}[k])(x-a)}}{(1+|k|(x-a))^{2}} \int_{a}^{x} d y y\|V(y)\|\|\omega(0, y)\|
$$


Since $\omega(0, y)$ is bounded and $V \in L_{1}^{1}\left(\mathbf{R}^{+}\right)$, from (5.17) we obtain

$$
\left\|K_{3}\right\| \leq \frac{c|k|^{2}(x-a)^{2} e^{(\operatorname{Im}[k])(x-a)}}{(1+|k|(x-a))^{2}}, \quad k \in \overline{\mathbf{C}^{+}}, \quad x \geq a
$$

for a generic constant $c$. Let us now estimate $K_{4}$ when $k \in \overline{\mathbf{C}^{+}}$and $x \geq a$. Letting

$$
\zeta(k, x):=e^{-(\operatorname{Im}[k])(x-a)}\|\omega(k, x)-\omega(0, x)\|,
$$

from (5.12) we get

$$
e^{-(\operatorname{Im}[k])(x-a)}|| K_{4}\left\|\leq \frac{1}{|k|} \int_{a}^{x} d y e^{-(\operatorname{Im}[k])(x-y)}|\sin k(x-y)|\right\| V(y) \| \zeta(k, y)
$$

Using the first estimate of (5.13) in (5.20) and the fact that $x \mapsto x /(1+x)$ is an increasing function of $x$ when $x \geq 0$, we obtain

$$
e^{-(\operatorname{Im}[k])(x-a)}\left\|K_{4}\right\| \leq \frac{c(x-a)}{(1+|k|(x-a))} \int_{a}^{x} d y\|V(y)\| \zeta(k, y) .
$$

Using (5.14), (5.16), (5.18), (5.19), and (5.21) in (5.8) we get

$$
\zeta(k, x) \leq \frac{c|k|^{2}(x-a)^{2}}{(1+|k|(x-a))^{2}}+\frac{c}{|k|} \frac{|k|(x-a)}{(1+|k|(x-a))} \int_{a}^{x} d y\|V(y)\| \zeta(k, y) .
$$

Setting

$$
\chi(k, x):=\frac{(1+|k|(x-a))^{2}}{c|k|^{2}(x-a)^{2}} \zeta(k, x)
$$

we can write $(5.22)$ as

$$
\chi(k, x) \leq 1+\frac{(1+|k|(x-a))}{|k|^{2}(x-a)} \int_{a}^{x} d y \| V(y)|| \frac{c|k|^{2}(y-a)^{2}}{(1+|k|(y-a))^{2}} \chi(k, y) .
$$

From (5.24) we obtain

$$
\chi(k, x) \leq 1+c \int_{a}^{x} d y y\|V(y)\| \chi(k, y)
$$

where we have used, for $0 \leq a \leq y \leq x$, the estimate

$$
\frac{|k|^{2}(y-a)^{2}}{(1+|k|(y-a))^{2}} \leq \frac{|k|(x-a)}{(1+|k|(x-a))} \frac{|k|(y-a)}{(1+|k|(y-a))} \leq \frac{|k|(x-a)}{(1+|k|(x-a))}|k| y
$$


based on the fact that $x \mapsto x /(1+x)$ is an increasing function of $x$ when $x \geq 0$. Applying Gronwall's lemma to (5.25) and using the fact that $V \in L_{1}^{1}\left(\mathbf{R}^{+}\right)$, we obtain $\chi(k, x) \leq c$ for some generic constant $c$, which is not necessarily equal to the generic constant $c$ in (5.25). Thus, using (5.19) and (5.23) in $\chi(k, x) \leq c$ we obtain (5.2).

Let us define

$$
P(k):=\left[\omega\left(-k^{*}, x\right)^{\dagger} ; f(k, x)\right]
$$

where we note that the Wronskian in (5.26) is independent of $x$, and hence with the help of (3.13) and (3.16) by evaluating that Wronskian at $x=a$ we get

$$
P(k)=f(0, a)^{\dagger} f^{\prime}(k, a)-f^{\prime}(0, a)^{\dagger} f(k, a) .
$$

Note that $P(k)$ has an analytic extension from $k \in \mathbf{R}$ to $k \in \mathbf{C}^{+}$because $f(k, a)$ and $f^{\prime}(k, a)$ possess that property as well. It is difficult to obtain useful information from (5.27) as $k \rightarrow 0$ because for $V \in L_{1}^{1}\left(\mathbf{R}^{+}\right)$we can only say that

$$
f(k, x)=f(0, x)+o(1), \quad f^{\prime}(k, x)=f^{\prime}(0, x)+o(1), \quad k \rightarrow 0 \text { in } \overline{\mathbf{C}^{+}} .
$$

In the proposition below we evaluate the small- $k$ asympotics of $P(k)$ by evaluating the Wronskian in (5.26) at $x=+\infty$. This result will be useful in evaluating the small- $k$ limit of the Jost matrix $J(k)$.

Proposition 5.2 If $V$ is selfadjoint and belongs to $L_{1}^{1}\left(\mathbf{R}^{+}\right)$, then the matrix $P(k)$ given in (5.26) satisfies

$$
P(k)=i k I_{n}+o(k), \quad k \rightarrow 0 \text { in } \overline{\mathbf{C}^{+}} .
$$

PROOF: We can evaluate the asymptotics of $\omega\left(-k^{*}, x\right)$ and $\omega^{\prime}\left(-k^{*}, x\right)$ as $x \rightarrow+\infty$ from (5.3). Furthermore, the asymptotics of $f(k, x)$ and $f^{\prime}(k, x)$ as $x \rightarrow+\infty$ are available from (3.1). Using those asymptotics in (5.26) we obtain

$$
P(k)=i k e^{i k a} f(0, a)^{\dagger}-e^{i k a} f^{\prime}(0, a)^{\dagger}-\int_{a}^{\infty} d y e^{i k y} \omega\left(-k^{*}, y\right)^{\dagger} V(y) .
$$


Let us break the right hand side in (5.30) into three terms and write

$$
P(k)=P_{1}(k)+P_{2}(k)+P_{3}(k),
$$

where we have defined

$$
\begin{gathered}
P_{1}(k):=i k e^{i k a} f(0, a)^{\dagger}-e^{i k a} f^{\prime}(0, a)^{\dagger} \\
P_{2}(k):=-\int_{a}^{\infty} d y e^{i k y}\left[\omega\left(-k^{*}, y\right)^{\dagger}-\omega(0, y)^{\dagger}\right] V(y), \\
P_{3}(k):=-\int_{a}^{\infty} d y e^{i k y} \omega(0, y)^{\dagger} V(y) .
\end{gathered}
$$

From (5.2) and the Lebesgue dominated convergence theorem, it follows that $P_{2}(k)=o(k)$ as $k \rightarrow 0$ in $\overline{\mathbf{C}^{+}}$whenever $V \in L_{1}^{1}\left(\mathbf{R}^{+}\right)$. Using (5.5) and (5.6) in (5.34) we have

$$
P_{3}(k)=f^{\prime}(0, a)^{\dagger}+i k\left[I_{n}+a f^{\prime}(0, a)^{\dagger}-f(0, a)^{\dagger}\right]+P_{4}(k),
$$

where we have defined

$$
P_{4}(k):=-\int_{a}^{\infty} d y\left[e^{i k y}-1-i k y\right] \omega(0, y)^{\dagger} V(y)
$$

Note that for any $z \in \overline{\mathbf{C}^{+}}$we have

$$
\left|e^{i z}-1\right| \leq c|z|, \quad\left|e^{i z}-1-i z\right| \leq \frac{c|z|^{2}}{1+|z|}
$$

where $c$ denotes a generic nonnegative constant independent of the complex number $z$, and hence the second inequality in (5.37) helps us to get $P_{4}(k)=o(k)$ as $k \rightarrow 0$ in $\overline{\mathbf{C}^{+}}$. Thus, using (5.31)-(5.36), we have

$$
P(k)=i k I_{n}+\left[i k e^{i k a}-i k\right] f(0, a)^{\dagger}+\left[I_{n}+i k a-e^{i k a}\right] f^{\prime}(0, a)^{\dagger}+o(k),
$$

and because of (5.37) each of the coefficients of $f(0, a)^{\dagger}$ and $f^{\prime}(0, a)^{\dagger}$ is $O\left(k^{2}\right)$ as $k \rightarrow 0$ in $\overline{\mathbf{C}^{+}}$.

Even though (5.28) does not provide much extraordinary information, the following theorem shows that $f^{\prime}(k, x)[f(k, x)]^{-1}$ is differentiable at $k=0$ at any fixed $x$ value 
where the matrix $f(0, x)$ is invertible. The results stated in the next theorem are the generalization to the matrix case of similar results in the scalar case $[2,3]$.

Theorem 5.3 Assume that $V$ in (1.1) is selfadjoint and belongs to $L_{1}^{1}\left(\mathbf{R}^{+}\right)$. If the constant matrix $f(0, a)$ is invertible, where $f(k, x)$ is the Jost solution appearing in (3.1), then, $f^{\prime}(k, a)[f(k, a)]^{-1}$ is differentiable at $k=0$ and we have

$$
f^{\prime}(k, a) f(k, a)^{-1}=f^{\prime}(0, a) f(0, a)^{-1}+i k\left[f(0, a)^{-1}\right]^{\dagger} f(0, a)^{-1}+o(k), \quad k \rightarrow 0 \text { in } \overline{\mathbf{C}^{+}}
$$

If instead, $f^{\prime}(0, a)$ is invertible at some $a \in \mathbf{R}^{+}$, then the Jost solution satisfies

$$
f(k, a) f^{\prime}(k, a)^{-1}=f(0, a) f^{\prime}(0, a)^{-1}-i k\left[f^{\prime}(0, a)^{-1}\right]^{\dagger} f^{\prime}(0, a)^{-1}+o(k), \quad k \rightarrow 0 \text { in } \overline{\mathbf{C}^{+}} .
$$

PROOF: In case $f(0, a)$ is invertible, by the continuity of the determinant of $f(k, a)$, we must have $f(k, a)$ invertible in $\overline{\mathbf{C}^{+}}$in the vicinity of $k=0$. Thus, from (5.27) we get

$$
f^{\prime}(k, a) f(k, a)^{-1}=\left[f(0, a)^{\dagger}\right]^{-1} f^{\prime}(0, a)^{\dagger}+\left[f(0, a)^{\dagger}\right]^{-1} P(k) f(k, a)^{-1} .
$$

Note that (3.19) implies that

$$
\left[f(0, a)^{\dagger}\right]^{-1} f^{\prime}(0, a)^{\dagger}=f^{\prime}(0, a) f(0, a)^{-1}
$$

Applying (5.29) in (5.40) and using (3.8) and (5.41), we obtain (5.38). In a similar way, (5.39) is proved.

Next, we express the Jost matrix $J(k)$ defined in (4.3) in terms of the Jost solution $f(k, x)$, the regular solutions $\varphi(k, x)$, the regular solution $\omega(k, x)$, and the matrix $P(k)$ appearing in (3.1), (3.6), (3.13), and (5.26), respectively.

Proposition 5.4 Assume that $V$ in (1.1) is selfadjoint and belongs to $L_{1}^{1}\left(\mathbf{R}^{+}\right)$. The Jost matrix can be written as

$$
J(k)=T_{1}(k)+T_{2}(k), \quad k \in \overline{\mathbf{C}^{+}},
$$


where we have defined

$$
\begin{gathered}
T_{1}(k):=-P\left(-k^{*}\right)^{\dagger} f(0, a)^{-1} \varphi(k, a), \\
T_{2}(k):=f\left(-k^{*}, a\right)^{\dagger}\left[f(0, a)^{-1}\right]^{\dagger}\left[\omega\left(-k^{*}, x\right)^{\dagger} ; \varphi(k, x)\right],
\end{gathered}
$$

and recall that the value of the Wronskian appearing in (5.44) is independent of $x$.

PROOF: Using (4.3) we can write $J(k)$ in terms of Wronskians evaluated at $x=a$ to get

$$
\begin{aligned}
J(k) & =\left.\left[f\left(-k^{*}, x\right)^{\dagger} ; \varphi(k, x)\right]\right|_{x=a} \\
& =f\left(-k^{*}, a\right)^{\dagger} \varphi^{\prime}(k, a)-f^{\prime}\left(-k^{*}, a\right)^{\dagger} \varphi(k, a) \\
& =T_{1}(k)+T_{2}(k)+T_{3}(k),
\end{aligned}
$$

where we have defined

$$
\begin{gathered}
T_{1}(k):=-\left(f^{\prime}\left(-k^{*}, a\right)^{\dagger} f(0, a)-f\left(-k^{*}, a\right)^{\dagger} f^{\prime}(0, a)\right) f(0, a)^{-1} \varphi(k, a), \\
T_{2}(k):=f\left(-k^{*}, a\right)^{\dagger}\left[f(0, a)^{-1}\right]^{\dagger}\left(f(0, a)^{\dagger} \varphi^{\prime}(k, a)-f^{\prime}(0, a)^{\dagger} \varphi(k, a)\right), \\
T_{3}(k):=f\left(-k^{*}, a\right)^{\dagger}\left[f(0, a)^{-1}\right]^{\dagger}\left(f^{\prime}(0, a)^{\dagger} f(0, a)-f(0, a)^{\dagger} f^{\prime}(0, a)\right) f(0, a)^{-1} \varphi(k, a) .
\end{gathered}
$$

Using (5.27) in (5.45) we see that $T_{1}(k)$ in (5.45) can equivalently be written as in (5.43). Using (3.13) in (5.46), we see that $T_{2}(k)$ in (5.46) can equivalently be written as

$$
\begin{aligned}
T_{2}(k) & =f\left(-k^{*}, a\right)^{\dagger}\left[f(0, a)^{-1}\right]^{\dagger}\left(\omega\left(-k^{*}, a\right)^{\dagger} \varphi^{\prime}(k, a)-\omega^{\prime}\left(-k^{*}, a\right)^{\dagger} \varphi(k, a)\right) \\
& =\left.f\left(-k^{*}, a\right)^{\dagger}\left[f(0, a)^{-1}\right]^{\dagger}\left[\omega\left(-k^{*}, x\right)^{\dagger} ; \varphi(k, x)\right]\right|_{x=a},
\end{aligned}
$$

which is equivalent to (5.44) because the Wronskian in (5.44) is independent of $x$ and can be evaluated at $x=a$. Finally, using (3.19) with $k=0$ in (5.47) we see that $T_{3}(k)=0$.

In the next theorem we evaluate the Wronskian $\left[\omega\left(-k^{*}, x\right)^{\dagger} ; \varphi(k, x)\right]$ appearing in (5.44).

Proposition 5.5 Assume that $V$ in (1.1) is selfadjoint and belongs to $L_{1}^{1}\left(\mathbf{R}^{+}\right)$. Then, the Wronskian appearing in (5.44) has the small-k asymptotics

$$
\left[\omega\left(-k^{*}, x\right)^{\dagger} ; \varphi(k, x)\right]=J(0)+O\left(k^{2}\right), \quad k \rightarrow 0 \text { in } \overline{\mathbf{C}^{+}},
$$


where $J(k)$ is the Jost matrix defined in (4.3).

PROOF: Since the value of the Wronskian in (5.48) is independent of $x$, we will evaluate its value at $x=0$. By writing

$$
\left[\omega\left(-k^{*}, x\right)^{\dagger} ; \varphi(k, x)\right]=\left[\omega\left(-k^{*}, x\right)^{\dagger}-\omega(0, x)^{\dagger} ; \varphi(k, x)\right]+\left[\omega(0, x)^{\dagger} ; \varphi(k, x)\right],
$$

from (3.6), (3.14), and (5.1) we see that the second Wronskian on the right side in (5.49), when $x=0$, yields

$$
\left.\left[\omega(0, x)^{\dagger} ; \varphi(k, x)\right]\right|_{x=0}=J(0) .
$$

Next, we evaluate at $x=0$ the value of the first Wronskian on the right side in (5.49). The $x$-derivative of that first Wronskian, with the help of (1.1) and (3.17), can be directly evaluated as

$$
\frac{d}{d x}\left[\omega\left(-k^{*}, x\right)^{\dagger}-\omega(0, x)^{\dagger} ; \varphi(k, x)\right]=k^{2} \omega(0, x)^{\dagger} \varphi(k, x) .
$$

Integrating (5.50) over the interval $[0, a]$, and then using (3.13) and (3.14), we obtain

$$
\left.\left[\omega\left(-k^{*}, x\right)^{\dagger}-\omega(0, x)^{\dagger} ; \varphi(k, x)\right]\right|_{x=0}=-k^{2} \int_{0}^{a} d y f(0, y)^{\dagger} \varphi(k, y) .
$$

Thus, we have the estimate in (5.48).

Using (5.4), (5.29), (5.42)-(5.44), and (5.48) we have the following conclusion.

Corollary 5.6 Assume that $V$ in (1.1) is selfadjoint and belongs to $L_{1}^{1}\left(\mathbf{R}^{+}\right)$. Then, the Jost matrix $J(k)$ appearing in (4.3) has the small-k behavior

$$
f(0, a)^{\dagger}\left[f\left(-k^{*}, a\right)^{\dagger}\right]^{-1} J(k)=J(0)-i k f(0, a)^{-1} \varphi(0, a)+o(k), \quad k \rightarrow 0 \text { in } \overline{\mathbf{C}^{+}},
$$

where $f(k, x)$ and $\varphi(k, x)$ are the Jost solution and the regular solution appearing in (3.1) and (3.6), respectively, and $a$ is any point where the matrix $f(0, a)$ is invertible.

In order to study the small- $k$ limit of $J(k)^{-1}$, we will next concentrate on the $O(k)$ term appearing in (5.51), namely $f(0, a)^{-1} \varphi(0, a)$. We use Ker $[J(0)]$ to denote the kernel of the matrix $J(0)$. 
Proposition 5.6 Assume that $V$ in (1.1) is selfadjoint and belongs to $L_{1}^{1}\left(\mathbf{R}^{+}\right)$. Then, the following are equivalent:

(a) The vector $u \in \mathbf{C}^{n}$ is an eigenvector of the zero-energy Jost matrix $J(0)$ with the zero eigenvalue, i.e. $u \in \operatorname{Ker}[J(0)]$.

(b) $\varphi^{\prime}(0,+\infty) u=0$, where $\varphi(k, x)$ is the regular solution to (1.1) appearing in (3.6).

(c) $\varphi(0, x) u$ is bounded for $x \in[0,+\infty)$.

PROOF: From (4.3) we see that

$$
J(0)=f(0, x)^{\dagger} \varphi^{\prime}(0, x)-f^{\prime}(0, x)^{\dagger} \varphi(0, x),
$$

where the quantity on the right side in (5.52) is independent of $x$. From (3.5) it follows that each column of $\varphi(0, x)$ is a linear combination of columns of $f(0, x)$ and $g(0, x)$. Hence, there exist constant $n \times n$ matrices $\alpha$ and $\beta$ such that

$$
\varphi(0, x)=f(0, x) \alpha+g(0, x) \beta, \quad x \in \mathbf{R}^{+} .
$$

From the $x$-derivative of $(5.53)$ we get

$$
\varphi^{\prime}(0, x)=f^{\prime}(0, x) \alpha+g^{\prime}(0, x) \beta, \quad x \in \mathbf{R}^{+} .
$$

Using (3.3) and (3.4) in (5.54) we see that

$$
\varphi^{\prime}(0,+\infty)=\beta .
$$

Inserting (5.53) and (5.54) on the right side of (5.52), evaluating the resulting expression as $x \rightarrow+\infty$, and using (3.3) and (3.4), we obtain

$$
J(0)=\beta .
$$

Thus, from (5.55) and (5.56) we conclude that

$$
J(0)=\varphi^{\prime}(0,+\infty)
$$


and hence the equivalence of (a) and (b) are established. Note that, from (3.3), (3.4), and (5.53) it follows that $\varphi(0, x) u$ is bounded if and only if $\beta u=0$, which happens if and only if $\varphi^{\prime}(0,+\infty) u=0$ as a result of (5.55). Thus, the equivalence of (b) and (c) is established.

Let us note that we can express $\varphi^{\prime}(0,+\infty)$ in (5.57) in another form. Letting $k \rightarrow 0$ in (3.7) we get

$$
\varphi(0, x)=A+B x+\int_{0}^{x} d y(x-y) V(y) \varphi(0, y),
$$

and from the $x$-derivative of $(5.58)$ we have

$$
\varphi^{\prime}(0, x)=B+\int_{0}^{x} d y V(y) \varphi(0, y),
$$

We know from (3.3)-(3.5) that $\varphi(0, x)$ can grow at most as $O(x)$ as $x \rightarrow+\infty$ and hence the integral in (5.59) exists as $x \rightarrow+\infty$, and from (5.57) and (5.59) we get

$$
J(0)=\varphi^{\prime}(0,+\infty)=B+\int_{0}^{\infty} d y V(y) \varphi(0, y) .
$$

Proposition 5.7 Assume that $V$ in (1.1) is selfadjoint and belongs to $L_{1}^{1}\left(\mathbf{R}^{+}\right)$. Then, for any vector $u$ in $\operatorname{Ker}[J(0)]$ there exists a unique vector $\xi$ in $\operatorname{Ker}\left[J(0)^{\dagger}\right]$ such that

$$
\varphi(0, x) u=f(0, x) \xi
$$

The map $u \mapsto \xi$ from $\operatorname{Ker}[J(0)]$ to $\operatorname{Ker}\left[J(0)^{\dagger}\right]$ is a bijection.

PROOF: By expressing $\varphi(0, x) u$ as in (3.5) we get

$$
\varphi(0, x) u=f(0, x) \xi+g(0, x) \eta,
$$

and hence $\varphi(0, x) u$ is bounded, equivalently as stated in Proposition 5.6, $u \in \operatorname{Ker}[J(0)]$, if and only if $\eta=0$. Thus, the mapping $u \mapsto \xi$ is identified with (5.44). Let us now show that $\xi \in \operatorname{Ker}\left[J(0)^{\dagger}\right]$. Using (5.1), we have

$$
J(0)^{\dagger} \xi=\left[B^{\dagger} f(0,0)-A^{\dagger} f^{\prime}(0,0)\right] \xi .
$$


On the other hand, from (5.44) and its derivative, with the help of (3.6), we see that

$$
f(0,0) \xi=\varphi(0,0) u=A u, \quad f^{\prime}(0,0) \xi=\varphi^{\prime}(0,0) u=B u
$$

Using (5.62) in (5.61) and imposing (1.12) we get $J(0)^{\dagger} \xi=0$. Thus, $\xi \in \operatorname{Ker}\left[J(0)^{\dagger}\right]$. Note that the map $u \mapsto \xi$ is a linear map from $\operatorname{Ker}[J(0)]$ into $\operatorname{Ker}\left[J(0)^{\dagger}\right]$ because, as seen from (5.60), we have

$$
\xi=f(0, a)^{-1} \varphi(0, a) u, \quad u \in \operatorname{Ker}[J(0)]
$$

The map $u \mapsto \xi$ from $\operatorname{Ker}[J(0)]$ to $\operatorname{Ker}\left[J(0)^{\dagger}\right]$ is one-to-one because it has zero kernel as seen by the following argument. If $\xi=0$ in (5.60), then we must have $\varphi(0, x) u=0$ and $\varphi^{\prime}(0, x) u=0$. In particular, at $x=0$ with the help of (3.6) we then have $A u=0$ and $B u=0$. We in turn get $A^{\dagger} A u=0$ and $B^{\dagger} B u=0$, and hence $\left(A^{\dagger} A+B^{\dagger} B\right) u=0$, which yields $u=0$ because of (1.13). Furthermore, $\operatorname{Ker}[J(0)]$ and $\operatorname{Ker}\left[J(0)^{\dagger}\right]$ have the same dimension, and hence the map $u \mapsto \xi$ from $\operatorname{Ker}[J(0)]$ to $\operatorname{Ker}\left[J(0)^{\dagger}\right]$ is a bijection.

\section{SMALL- $k$ BEHAVIOR OF $J(k)^{-1}$ AND OF $S(k)$}

In this section we establish the small- $k$ asymptotics of the Jost matrix $J(k)$, its inverse $J(k)^{-1}$, and the scattering matrix $S(k)$. As we will see, $J(k)$ is continuous at $k=0, J(k)^{-1}$ has an $O(1 / k)$ singularity at $k=0$ if $J(0)$ has a zero eigenvalue, $J(k)$ is continuous at $k=0$ if zero is not an eigenvalue of $J(0)$, and that $S(k)$ is continuous at $k=0$ whether or not zero is an eigenvalue of $J(0)$.

In order to analyze the small- $k$ behavior of $J(k)^{-1}$, we will analyze (5.51). Let us write $(5.51)$ as

$$
F(k)=J(0)-i k R+o(k), \quad k \rightarrow 0 \text { in } \overline{\mathbf{C}^{+}},
$$

where we have defined

$$
F(k):=f(0, a)^{\dagger}\left[f\left(-k^{*}, a\right)^{-1}\right]^{\dagger} J(k), \quad R:=f(0, a)^{-1} \varphi(0, a) .
$$

We will equivalently analyze the behavior of $F(k)^{-1}$ as $k \rightarrow 0 \in \overline{\mathbf{C}^{+}}$. As we have seen in Proposition 5.7, the restriction of $R$ to $\operatorname{Ker}[J(0)]$ yields an invertible map. Among the $n$ 
eigenvalues of $J(0)$, let us assume that the zero eigenvalue has geometric multiplicity $\mu$ with a possibly larger algebraic multiplicity $\nu$. In other words, $J(0)$ has $\mu$ linearly independent eigenvectors corresponding to the zero eigenvalue. Thus, $J(0) v=0$ if and only if $v \in \mathbf{C}^{n}$ is such an eigenvector, in which case we have $R v \neq 0$.

Let us choose a Jordan basis [10] for the matrix $J(0)$ as follows. Assume that there are $\kappa$ Jordan chains and hence $\kappa$ blocks in the Jordan canonical form of $J(0)$. Let us use the index $\alpha$ for $\alpha=1, \ldots, \kappa$ to identify the Jordan chains and assume that the $\alpha$ th chain consists of $n_{\alpha}$ vectors $u_{\alpha j}$ for $j=1, \ldots, n_{\alpha}$. Let us use $\lambda_{\alpha}$ to denote the eigenvalue of $J(0)$ associated with the $\alpha$ th chain, where the eigenvalues may be repeated. We have

$$
\left\{\begin{array}{l}
{\left[J(0)-\lambda_{\alpha}\right] u_{\alpha 1}=0,} \\
{\left[J(0)-\lambda_{\alpha}\right] u_{\alpha j}=u_{\alpha(j-1)}, \quad j=2, \ldots, n_{\alpha},}
\end{array}\right.
$$

and hence $u_{\alpha 1}$ is an eigenvector and $u_{\alpha j}$ for $j=2, \ldots, n_{\alpha}$ are the generalized eigenvectors.

Since we assume the zero eigenvalue has geometric multiplicity $\mu$, without loss of generality we let $\lambda_{\alpha}=0$ for $\alpha=1, \ldots, \mu$ and $\lambda_{\alpha} \neq 0$ for $\alpha=\mu+1, \ldots, \kappa$. We order the vectors in the Jordan basis according to the rule that $u_{\alpha j}$ comes before $u_{\beta s}$ if and only if $\alpha<\beta$ or $\alpha=\beta$ and $j<s$. Thus, $\left\{u_{11}, u_{21}, \ldots, u_{\mu 1}\right\}$ forms a basis for $\operatorname{Ker}[J(0)]$, and our Jordan basis is given by the ordered set $\left\{u_{\alpha j}\right\}$, i.e.

$$
\left\{u_{11}, u_{12}, \ldots, u_{1 n_{1}}, u_{21}, u_{22}, \ldots, u_{2 n_{2}}, \ldots, u_{\kappa 1}, u_{\kappa 2}, \ldots, u_{\kappa n_{\kappa}}\right\}
$$

The corresponding adjoint Jordan basis $\left\{v_{\alpha j}\right\}$ satisfies $v_{\alpha j}^{\dagger} u_{\rho t}=\delta_{\alpha \rho} \delta_{j t}$, with $\delta_{j t}$ denoting the Kronecker delta, and the indices $\alpha$ and $\rho$ referring to the Jordan blocks. The vectors $v_{\alpha j}$ satisfy

$$
\left\{\begin{array}{l}
{\left[J(0)^{\dagger}-\lambda_{\alpha}^{*}\right] v_{\alpha n_{\alpha}}=0,} \\
{\left[J(0)^{\dagger}-\lambda_{\alpha}^{*}\right] v_{\alpha j}=u_{\alpha(j+1)}, \quad j=1, \ldots, n_{\alpha}-1 .}
\end{array}\right.
$$

Thus, $\left\{v_{1 n_{1}}, v_{2 n_{2}}, \ldots, v_{\mu n_{\mu}}\right\}$ forms a basis for Ker $\left[J(0)^{\dagger}\right]$. The adjoint Jordan basis is the ordered set $\left\{v_{\alpha j}\right\}$, i.e.

$$
\left\{v_{11}, v_{12}, \ldots, v_{1 n_{1}}, v_{21}, v_{22}, \ldots, v_{2 n_{2}}, \ldots, v_{\kappa 1}, v_{\kappa 2}, \ldots, v_{\kappa n_{\kappa}}\right\}
$$


Let $\mathcal{S}$ denote the matrix whose columns are given by the elements of the ordered set $\left\{u_{\alpha j}\right\}$ in (6.4). Then, $\mathcal{S}^{-1}$ is exactly the matrix whose rows are given by the elements of the ordered set $\left\{v_{\alpha j}^{\dagger}\right\}$, with the ordering given in (6.6). Thus, the Jordan canonical form of $J(0)$ is given by

$$
\mathcal{S}^{-1} J(0) \mathcal{S}=\oplus_{\alpha=1}^{\kappa} J_{n_{\alpha}}\left(\lambda_{\alpha}\right),
$$

where $J_{n_{\alpha}}\left(\lambda_{\alpha}\right)$ is the $n_{\alpha} \times n_{\alpha}$ Jordan block with $\lambda_{\alpha}$ appearing in the diagonal entries and one in the superdiagonal entries. Since the first $\mu$ Jordan blocks $J_{n_{\alpha}}\left(\lambda_{\alpha}\right)$ are associated with the zero eigenvalue and the remaining $(n-\mu)$ blocks are associated with nonzero eigenvalues, each $J_{n_{\alpha}}\left(\lambda_{\alpha}\right)$ is an $n_{\alpha} \times n_{\alpha}$ matrix given by

$$
\begin{aligned}
J_{n_{\alpha}}\left(\lambda_{\alpha}\right)=\left[\begin{array}{cccccc}
0 & 1 & 0 & \ldots & 0 & 0 \\
0 & 0 & 1 & \ldots & 0 & 0 \\
\vdots & \vdots & \vdots & \ddots & \vdots & \vdots \\
0 & 0 & 0 & \ldots & 0 & 1 \\
0 & 0 & 0 & \ldots & 0 & 0
\end{array}\right], \quad \alpha=1, \ldots, \mu, \\
J_{n_{\alpha}}\left(\lambda_{\alpha}\right)=\left[\begin{array}{cccccc}
\lambda_{\alpha} & 1 & 0 & \ldots & 0 & 0 \\
0 & \lambda_{\alpha} & 1 & \ldots & 0 & 0 \\
\vdots & \vdots & \vdots & \ddots & \vdots & \vdots \\
0 & 0 & 0 & \ldots & \lambda_{\alpha} & 1 \\
0 & 0 & 0 & \ldots & 0 & \lambda_{\alpha}
\end{array}\right], \quad \alpha=\mu+1, \ldots, \kappa .
\end{aligned}
$$

Let us use a tilde to denote the transformation via $\mathcal{S}$, i.e. $\tilde{M}:=\mathcal{S}^{-1} M \mathcal{S}$ for any $n \times n$ matrix $M$. Let us apply this transformation on the matrix $F(k)$ appearing in (6.1) and (6.2). Then (6.1) yields

$$
\tilde{F}(k)=\tilde{J}(0)-i k \tilde{R}+o(k), \quad k \rightarrow 0 \text { in } \overline{\mathbf{C}^{+}} .
$$

By inspecting (6.7)-(6.10) we see that there are exactly $\mu$ columns of $\tilde{F}(k)$ behaving as $O(k)$ as $k \rightarrow 0$ and each of the remaining $(n-\mu)$ column vectors contains at least one entry that has a nonzero limit as $k \rightarrow 0$.

Our next goal is to move all the entries with 1 appearing in the superdiagonal in the first $\mu$ Jordan blocks in (6.8) and collect all those entries into the $(\nu-\mu)$ identity matrix 
$I_{\nu-\mu}$. Recall that $\nu$ and $\mu$ correspond to the algebraic and geometric multiplicities of the zero eigenvalue of $J(0)$, and hence there are exactly $(\nu-\mu)$ such entries to move. Such a movement will be accomplished by first permuting some of the first $\nu$ columns in $\tilde{J}(0)$ and then by permuting some of the first $\nu$ rows of the resulting matrix. The permutations among the first $\nu$ columns can be described by a matrix postmultiplying $\tilde{J}(0)$ and we use $P_{1}$ to denote that matrix. On the other hand, the permutations among the first $\nu$ rows can be described by a matrix premultiplying $\tilde{J}(0)$ and we use $P_{2}$ to denote that matrix. Thus, the matrix $P_{2} \tilde{J}(0) P_{1}$ will be given by

$$
P_{2} \tilde{J}(0) P_{1}=\operatorname{diag}\left\{0_{\mu}, I_{\nu-\mu}, J_{n_{\mu}+1}\left(\lambda_{\mu+1}\right), \ldots, J_{n_{\kappa}}\left(\lambda_{\kappa}\right)\right\}
$$

where $0_{\mu}$ denotes the $\mu \times \mu$ zero matrix. Since $P_{1}$ and $P_{2}$ affect only the first $\nu$ columns and $\nu$ rows, respectively, they have the form

$$
P_{1}=\left[\begin{array}{cc}
\Pi_{1} & 0 \\
0 & I_{n-\nu}
\end{array}\right], \quad P_{2}=\left[\begin{array}{cc}
\Pi_{2} & 0 \\
0 & I_{n-\nu}
\end{array}\right],
$$

for some permutation matrices $\Pi_{1}$ and $\Pi_{2}$.

Formally speaking, the matrix $\Pi_{1}$ describes the permutation $\pi_{1}$ given by

$$
\pi_{1}:(1, \ldots, \nu) \mapsto\left(q_{1}, \ldots, q_{\nu}\right)
$$

where

$$
q_{\tau}= \begin{cases}n_{1}+\cdots+n_{\tau-1}+1, & \tau=1, \ldots, \mu, \\ \tau-\mu+\alpha, & \tau=\mu+1, \ldots, \nu,\end{cases}
$$

and $\alpha \in\{1, \ldots, \mu\}$ is the unique integer such that, for given $\tau$ and $\mu$,

$$
n_{1}+n_{2}+\cdots+n_{\alpha-1}-\alpha+j=\tau-\mu
$$

for some $j \in\left\{2, \ldots, n_{\alpha}\right\}$. Note that, since $n_{\alpha} \geq 1$, the quantity $n_{1}+n_{2}+\cdots+n_{\alpha-1}-\alpha$ is a nondecreasing function of $\alpha$.

Similarly, $\Pi_{2}$ is related to the permutation $\pi_{2}$ given by

$$
\pi_{2}:(1, \ldots, \nu) \mapsto\left(\sigma_{1}, \ldots, \sigma_{\nu}\right)
$$


where

$$
\sigma_{\alpha}=\left\{\begin{array}{l}
n_{1}+\cdots+n_{\alpha}, \quad \alpha=1, \ldots, \mu, \\
\alpha-\mu+\rho-1, \quad \alpha=\mu+1, \ldots, \nu,
\end{array}\right.
$$

and $\rho \in\{1, \ldots, \mu\}$ is the unique integer such that, for given $\alpha$ and $\mu$

$$
n_{1}+n_{2}+\cdots+n_{\rho-1}-\rho+s=\alpha-\mu
$$

for some $s \in\left\{2, \ldots, n_{\rho}\right\}$. To implement these permutations we let $\hat{e}_{j}$ for $j=1, \ldots, \nu$ denote the column vectors of the standard basis in $\mathbf{C}^{\nu}$ and let $\Pi_{1}$ be the $\nu \times \nu$ permutation matrix whose $j$ th column vector is $\hat{e}_{q_{j}}$, and let $\Pi_{2}$ be the $\nu \times \nu$ permutation matrix whose $k$ th row vector is $\hat{e}_{\sigma_{k}}^{\dagger}$. Now observe that, if $M$ is any $\nu \times \nu$ matrix, then the matrix $\Pi_{2} M \Pi_{1}$ can be thought of as being obtained from $M$ by a permutation of the columns according to $\pi_{1}$ and a permutation of the rows according to $\pi_{2}$.

Let us now return to the matrix $F(k)$ defined in (6.2). By first putting it into the Jordan canonical form $\tilde{F}(k)$ and then by applying $P_{1}$ and $P_{2}$ on the first $\nu$ columns and rows of $\tilde{F}(k)$, we form the matrix $\mathcal{Z}(k)$ defined as

$$
\mathcal{Z}(k):=\left[\begin{array}{ll}
\mathcal{A}(k) & \mathcal{B}(k) \\
\mathcal{C}(k) & \mathcal{D}(k)
\end{array}\right]:=P_{2} \tilde{F}(k) P_{1}=P_{2} \mathcal{S}^{-1} F(k) \mathcal{S} P_{1},
$$

where $\mathcal{A}(k)$ has size $\mu \times \mu, \mathcal{D}(k)$ has size $(n-\mu) \times(n-\mu), \mathcal{A}(k)$ coincides with the submatrix of $\tilde{F}(k)$ consisting of the entries in columns $\alpha 1$ and rows $s n_{s}$, where $\alpha=1, \ldots, \mu$ and $s=1, \ldots, \mu$. The procedure of going from $F(k)$ to $\mathcal{Z}(k)$ is similar to the procedure described on pp. 4638-4639 of [4], where the mappings $P_{1}$ and $P_{2}$ were also used.

The small- $k$ limits of the block entries in the matrix $\mathcal{Z}(k)$ are described in the following theorem.

Theorem 6.1 Assume that $V$ in (1.1) is selfadjoint and belongs to $L_{1}^{1}\left(\mathbf{R}^{+}\right)$. Then, the asymptotics as $k \rightarrow 0$ in $\overline{\mathbf{C}^{+}}$of the matrices $\mathcal{A}(k), \mathcal{B}(k), \mathcal{C}(k), \mathcal{D}(k)$ appearing in (6.12) are given by

$$
\mathcal{A}(k)=k \mathcal{A}_{1}+o(k), \quad \mathcal{B}(k)=k \mathcal{B}_{1}+o(k), \quad \mathcal{C}(k)=k \mathcal{C}_{1}+o(k), \quad \mathcal{D}(k)=\mathcal{D}_{0}+O(k)
$$


where $\mathcal{A}_{1}, \mathcal{B}_{1}, \mathcal{C}_{1}, \mathcal{D}_{0}$ are constant matrices, and furthermore $\mathcal{A}_{1}$ and $\mathcal{D}_{0}$ are invertible.

PROOF: The proof for the expansions in (6.13) is similar to the proof of Proposition 4.4 of [4]. The invertibility of $\mathcal{D}_{0}$ follows from the fact that it consists of invertible blocks and is given by

$$
\mathcal{D}_{0}=\operatorname{diag}\left\{I_{\nu-\mu}, J_{n_{\mu}+1}\left(\lambda_{n_{\mu}+1}\right), \ldots, J_{n_{\kappa}}\left(\lambda_{n_{\kappa}}\right)\right\},
$$

where $I_{\nu-\mu}$ is the identity matrix of size $(\nu-\mu)$ with $\mu$ and $\nu$ denoting the respective geometric and algebraic multiplicities of the zero eigenvalue of $J(0)$, and the $J_{n_{\alpha}}\left(\lambda_{\alpha}\right)$ are the Jordan block matrices appearing in (6.7) corresponding to the nonzero eigenvalues for $\alpha=\mu+1, \ldots, \kappa$. From (6.1) and (6.10), as the $(s, j)$-entry of the matrix $\mathcal{A}_{1}$ we get

$$
\left(\mathcal{A}_{1}\right)_{s j}=-i v_{s n_{s}}^{\dagger} R u_{j 1},
$$

where $R$ is the matrix appearing in (6.1) and (6.2). By (5.63) and Proposition 5.7 we know that $R$ acts as an invertible map from $\operatorname{Ker}[J(0)]$ to $\operatorname{Ker}\left[J(0)^{\dagger}\right]$. Recall from (6.3) and (6.5) that $\left\{u_{11}, u_{21}, \ldots, u_{\mu j}\right\}$ is the Jordan basis for $\operatorname{Ker}[J(0)]$ and $\left\{v_{1 n_{1}}, v_{2 n_{2}}, \ldots, v_{\mu n_{\mu}}\right\}$ is the Jordan basis for $\operatorname{Ker}[J(0) \dagger]$. Thus, the matrix $\mathcal{A}_{1}$ is nothing but, apart from the factor $(-i)$, the matrix representation of the invertible map $R$ with respect to the Jordan basis and the adjoint Jordan basis. Thus, $\mathcal{A}_{1}$ is invertible.

Theorem 6.2 Assume that $V$ in (1.1) is selfadjoint and belongs to $L_{1}^{1}\left(\mathbf{R}^{+}\right)$. Then, the asymptotics as $k \rightarrow 0$ in $\overline{\mathbf{C}^{+}}$of the inverse of the matrix $\mathcal{Z}(k)$ appearing in (6.12) is given by

$$
\mathcal{Z}(k)^{-1}=\left[\begin{array}{cc}
\frac{1}{k} \mathcal{A}_{1}^{-1}\left[I_{\mu}+o(1)\right] & -\mathcal{A}_{1}^{-1} \mathcal{B}_{1} \mathcal{D}_{0}^{-1}+o(1) \\
-\mathcal{D}_{0}^{-1} \mathcal{C}_{1} \mathcal{A}_{1}^{-1}+o(1) & \mathcal{D}_{0}^{-1}+O(k)
\end{array}\right],
$$

where $\mathcal{A}_{1}, \mathcal{C}_{1}$, and $\mathcal{D}_{0}$ are the constant matrices appearing in (6.13) and the invertibility of $\mathcal{A}_{1}$ and $\mathcal{D}_{0}$ is assured in Theorem 6.1.

PROOF: The proof is exactly the same as the proof of Proposition 4.5(i) of [4]. We will use the decomposition formula $[4,10]$

$$
\left[\begin{array}{cc}
I_{\mu} & -\mathcal{B D}^{-1} \\
0 & I_{n-\mu}
\end{array}\right]\left[\begin{array}{cc}
\mathcal{A} & \mathcal{B} \\
\mathcal{C} & \mathcal{D}
\end{array}\right]\left[\begin{array}{cc}
I_{\mu} & 0 \\
-\mathcal{D}^{-1} \mathcal{C} & I_{n-\mu}
\end{array}\right]=\left[\begin{array}{cc}
\mathcal{A}-\mathcal{B D}^{-1} \mathcal{C} & 0 \\
0 & \mathcal{D}
\end{array}\right]
$$


Thus, as seen from $(6.15)$ for the matrix $\mathcal{Z}(k)$ defined in $(6.12)$ we have

$$
\mathcal{Z}^{-1}=\left[\begin{array}{cc}
\mathcal{A} & \mathcal{B} \\
\mathcal{C} & \mathcal{D}
\end{array}\right]^{-1}=\left[\begin{array}{cc}
I_{\mu} & 0 \\
-\mathcal{D}^{-1} \mathcal{C} & I_{n-\mu}
\end{array}\right]\left[\begin{array}{cc}
\left(\mathcal{A}-\mathcal{B D}^{-1} \mathcal{C}\right)^{-1} & 0 \\
0 & \mathcal{D}^{-1}
\end{array}\right]\left[\begin{array}{cc}
I_{\mu} & -\mathcal{B D}^{-1} \\
0 & I_{n-\mu}
\end{array}\right]
$$

or equivalently

$$
\mathcal{Z}(k)^{-1}=\left[\begin{array}{cc}
\left(\mathcal{A}-\mathcal{B D}^{-1} \mathcal{C}\right)^{-1} & -\left(\mathcal{A}-\mathcal{B D}^{-1} \mathcal{C}\right)^{-1} \mathcal{B D}^{-1} \\
-\mathcal{D}^{-1} \mathcal{C}\left(\mathcal{A}-\mathcal{B D}^{-1} \mathcal{C}\right)^{-1} & \mathcal{D}^{-1} \mathcal{C}\left(\mathcal{A}-\mathcal{B D}^{-1} \mathcal{C}\right)^{-1} \mathcal{B D}^{-1}+\mathcal{D}^{-1}
\end{array}\right]
$$

Finally, using (6.13) in (6.16) and the fact that $\mathcal{A}_{1}$ and $\mathcal{D}_{0}$ are invertible, we get (6.14). Let us note that we have written $(1 / k) \mathcal{A}_{1}^{-1}\left[I_{\mu}+o(1)\right]$ in the top left block in (6.14) whereas that term was written as $(1 / k) \mathcal{A}_{1}^{-1}+o(1 / k)$ in [4]. The two expressions are certainly equivalent because we can always premultiply the $o(1 / k)$-term by $\mathcal{A}_{1}^{-1} \mathcal{A}_{1}$.

We are now ready to evaluate the small- $k$ limit of the Jost matrix $J(k)$ defined in (4.3), its inverse $J(k)^{-1}$, and the scattering matrix $S(k)$ defined in (4.6). From (6.2) and (6.12) we see that the Jost matrix $J(k)$ is given by

$$
J(k)=f\left(-k^{*}, a\right)^{\dagger}\left[f(0, a)^{\dagger}\right]^{-1} \mathcal{S} P_{2}^{-1} \mathcal{Z}(k) P_{1}^{-1} \mathcal{S}^{-1},
$$

where the small- $k$ limit will be evaluated with the help of (3.8), (6.12), and (6.13). On the other hand, from (6.17) we get

$$
J(k)^{-1}=\mathcal{S} P_{1} \mathcal{Z}(k)^{-1} P_{2} \mathcal{S}^{-1} f(0, a)^{\dagger}\left[f\left(-k^{*}, a\right)^{\dagger}\right]^{-1}
$$

where the small- $k$ limit will be evaluated with the help of (3.8) and (6.14). Thus, using (6.17) and (6.18) in (4.6) we obtain

$$
S(k)=-f(k, a)^{\dagger}\left[f(0, a)^{\dagger}\right]^{-1} \mathcal{S} P_{2}^{-1} \mathcal{Z}(-k) \mathcal{Z}(k)^{-1} P_{2} \mathcal{S}^{-1} f(0, a)^{\dagger}\left[f(-k, a)^{\dagger}\right]^{-1} .
$$

In evaluating the small- $k$ limits of $J(k), J(k)^{-1}$, and $S(k)$, we will use a consequence of (3.8), namely for $k \rightarrow 0$ in $\overline{\mathbf{C}^{+}}$we have

$$
f(0, a)^{\dagger}\left[f\left(-k^{*}, a\right)^{\dagger}\right]^{-1}=I_{n}+o(1), \quad f(k, a)^{\dagger}\left[f(0, a)^{\dagger}\right]^{-1}=I_{n}+o(1) .
$$


Theorem 6.3 Assume that $V$ in (1.1) is selfadjoint and belongs to $L_{1}^{1}\left(\mathbf{R}^{+}\right)$. Then, as $k \rightarrow 0$ in $\overline{\mathbf{C}^{+}}$the Jost matrix $J(k)$ has the behavior

$$
J(k)=\mathcal{S} P_{2}^{-1}\left[\begin{array}{cc}
k \mathcal{A}_{1}+o(k) & k \mathcal{B}_{1} \mathcal{A}_{1}+o(k) \\
k \mathcal{C}_{1}+o(k) & \mathcal{D}_{0}+o(1)
\end{array}\right] P_{1} \mathcal{S}^{-1}
$$

the inverse Jost matrix $J(k)^{-1}$ has the behavior, as $k \rightarrow 0$ in $\overline{\mathbf{C}^{+}}$,

$$
J(k)^{-1}=\mathcal{S} P_{1}\left[\begin{array}{cc}
\frac{1}{k} \mathcal{A}_{1}^{-1}\left[I_{\mu}+o(1)\right] & -\mathcal{A}_{1}^{-1} \mathcal{B}_{1} \mathcal{D}_{0}^{-1}+o(1) \\
-\mathcal{D}_{0}^{-1} \mathcal{C}_{1} \mathcal{A}_{1}^{-1}+o(1) & \mathcal{D}_{0}^{-1}+o(1)
\end{array}\right] P_{2} \mathcal{S}^{-1}
$$

and the scattering matrix $S(k)$ defined in (4.6) is continuous at $k=0$ and we have $S(k)=$ $S(0)+o(1)$ as $k \rightarrow 0$ in $\mathbf{R}$ with

$$
S(0)=\mathcal{S} P_{2}^{-1}\left[\begin{array}{cc}
I_{\mu} & 0 \\
2 \mathcal{C}_{1} \mathcal{A}_{1}^{-1} & -I_{n-\mu}
\end{array}\right] P_{2} \mathcal{S}^{-1}
$$

where $\mathcal{A}_{1}, \mathcal{B}_{1}, \mathcal{C}_{1}$, and $\mathcal{D}_{0}$ are the matrices appearing in (6.13), $\mu$ is the geometric multiplicity of the zero eigenvalue of the zero-energy Jost matrix $J(0), P_{1}$ and $P_{2}$ are the permutation operators appearing in (6.11), and $\mathcal{S}$ is the matrix appearing in (6.7).

PROOF: Using (6.13) and (6.20) in (6.17) we get (6.21). Using (6.14) and (6.20) in (6.18) we obtain (6.22). Finally, using (6.12)-(6.14) we obtain

$$
\mathcal{Z}(-k) \mathcal{Z}(k)^{-1}=\left[\begin{array}{cc}
-I_{\mu}+o(1) & O(k) \\
-2 \mathcal{C}_{1} \mathcal{A}_{1}^{-1}+o(1) & I_{n-\mu}+O(k)
\end{array}\right], \quad k \rightarrow 0 \text { in } \mathbf{R} .
$$

Then, using (6.24) and (6.20) in (6.19) we get (6.23) and $S(k)=S(0)+o(1)$ as $k \rightarrow 0$ in

\section{R.}

\section{EXAMPLES}

In this section, we will check the validity of our formula (6.23) for some selfadjoint boundary conditions.

Example 7.1 (The $\delta^{\prime}$ boundary condition) As our first example, let us use the $3 \times 3$ versions of $A$ and $B$ from p. S116 of [25], with $A$ and $B$ satisfying (1.11)-(1.13) and given 
by

$$
A=\left[\begin{array}{ccc}
1 & 0 & -a \\
-1 & 1 & 0 \\
0 & -1 & 0
\end{array}\right], \quad B=\left[\begin{array}{ccc}
0 & 0 & -1 \\
0 & 0 & -1 \\
0 & 0 & -1
\end{array}\right]
$$

where $a$ is a real parameter and the potential $V$ is zero. From (4.3) with $f(k, x)=e^{i k x} I_{3}$, we obtain $J(k)$, and from (4.6) we obtain the scattering matrix $S(k)$, and we have

$$
J(k)=\left[\begin{array}{ccc}
-i k & 0 & -1+i a k \\
i k & -i k & -1 \\
0 & i k & -1
\end{array}\right], \quad S(k)=\left[\begin{array}{ccc}
\frac{i+a k}{3 i+a k} & \frac{-2 i}{3 i+a k} & \frac{-2 i}{3 i+a k} \\
\frac{-2 i}{3 i+a k} & \frac{i+a k}{3 i+a k} & \frac{-2 i}{3 i+a k} \\
\frac{-2 i}{3 i+a k} & \frac{-2 i}{3 i+a k} & \frac{i+a k}{3 i+a k}
\end{array}\right] .
$$

From (7.1) we see that $S(k)$ is continuous at $k=0$ and

$$
S(0)=\left[\begin{array}{ccc}
\frac{1}{3} & -\frac{2}{3} & -\frac{2}{3} \\
-\frac{2}{3} & \frac{1}{3} & -\frac{2}{3} \\
-\frac{2}{3} & -\frac{2}{3} & \frac{1}{3}
\end{array}\right]
$$

On the other hand, we see that $J(0)$ is given by

$$
J(0)=\left[\begin{array}{ccc}
0 & 0 & -1 \\
0 & 0 & -1 \\
0 & 0 & -1
\end{array}\right]
$$

and hence its eigenvalues are $\lambda_{1}=0, \lambda_{2}=0$, and $\lambda_{3}=-1$, with respective eigenvectors

$$
u_{11}=\left[\begin{array}{l}
1 \\
0 \\
0
\end{array}\right], \quad u_{21}=\left[\begin{array}{l}
0 \\
1 \\
0
\end{array}\right], \quad u_{31}=\left[\begin{array}{l}
1 \\
1 \\
1
\end{array}\right] \text {. }
$$

Thus, in the notation of Section 6 we have $n_{1}=1, n_{2}=1$, and $n_{3}=1$, with $\kappa=3$. Our permutation operators $P_{1}$ and $P_{2}$ are given by $P_{1}=I_{3}$ and $P_{2}=I_{3}$, and the matrix $\mathcal{S}$ appearing in (6.7) and the matrix $\mathcal{Z}(k)$ appearing in (6.12) are given by

$$
\mathcal{S}=\left[\begin{array}{lll}
1 & 0 & 1 \\
0 & 1 & 1 \\
0 & 0 & 1
\end{array}\right], \quad \mathcal{Z}(k)=\left[\begin{array}{ccc}
-i k & -i k & (a-2) i k \\
i k & -2 i k & -i k \\
0 & i k & -1+i k
\end{array}\right]
$$


From (7.3), with the help of (6.13) we get

$$
\mathcal{A}_{1}=\left[\begin{array}{cc}
-i & -i \\
i & -2 i
\end{array}\right], \quad \mathcal{B}_{1}=\left[\begin{array}{c}
(a-2) i \\
-i
\end{array}\right], \quad \mathcal{C}_{1}=\left[\begin{array}{ll}
0 & i
\end{array}\right], \quad \mathcal{D}_{0}=[-1] .
$$

Since $\mu=\nu=2$ and $n=3$ in our example, we evaluate (6.23) and confirm that the right hand side in (6.23) coincides with the matrix in (7.2).

Example 7.2 (The Kirchhoff boundary condition) The procedure in this example is similar to that of Example 7.1. We use the $3 \times 3$ versions of $A$ and $B$ from p. S117 of [25]. Using $V=0$ and

$$
A=\left[\begin{array}{lll}
0 & 0 & 1 \\
0 & 0 & 1 \\
0 & 0 & 1
\end{array}\right], \quad B=\left[\begin{array}{ccc}
-1 & 0 & 0 \\
1 & -1 & 0 \\
0 & 1 & 0
\end{array}\right]
$$

we obtain

$$
J(k)=\left[\begin{array}{ccc}
-1 & 0 & -i k \\
1 & -1 & -i k \\
0 & 1 & -i k
\end{array}\right], \quad S(k)=S(0)=\left[\begin{array}{ccc}
-\frac{1}{3} & \frac{2}{3} & \frac{2}{3} \\
\frac{2}{3} & -\frac{1}{3} & \frac{2}{3} \\
\frac{2}{3} & \frac{2}{3} & -\frac{1}{3}
\end{array}\right] .
$$

On the other hand, we have $J(0)$ given by

$$
J(0)=\left[\begin{array}{ccc}
-1 & 0 & 0 \\
1 & -1 & 0 \\
0 & 1 & 0
\end{array}\right] .
$$

The eigenvalues of $J(0)$ are $\lambda_{1}=0$ with $n_{1}=1$ and $\lambda_{2}=-1$ with $n_{2}=2$. We further have

$$
\begin{gathered}
\mu=\nu=1, \quad n=3, \quad P_{1}=P_{2}=I_{3}, \\
\mathcal{S}=\left[\begin{array}{ccc}
0 & 0 & -1 / \sqrt{2} \\
0 & -1 / \sqrt{2} & 1 / \sqrt{2} \\
1 & 1 / \sqrt{2} & 0
\end{array}\right], \quad \mathcal{Z}(k)=\left[\begin{array}{ccc}
-3 i k & -3 i k / \sqrt{2} & 0 \\
2 \sqrt{2} i k & -1+2 i k & 1 \\
\sqrt{2} i k & i k & -1
\end{array}\right], \\
\mathcal{A}_{1}=[-3 i], \quad \mathcal{B}_{1}=\left[\begin{array}{ll}
-3 i / \sqrt{2} & 0
\end{array}\right], \quad \mathcal{C}_{1}=\left[\begin{array}{c}
2 \sqrt{2} i \\
\sqrt{2} i
\end{array}\right], \quad \mathcal{D}_{0}=\left[\begin{array}{cc}
-1 & 1 \\
0 & -1
\end{array}\right] .
\end{gathered}
$$


Using the information in (7.5)-(7.7) in (6.23) we can verify that the right side in (6.23) coincides with $S(0)$ given in (7.4).

Example 7.3 (The XOR gate boundary condition) Recall that the XOR gate is a digital logic gate implementing an exclusive disjunction, yielding false if the two inputs agree and yielding true if the inputs disagree. The procedure in this example is similar to that of Example 7.1. We use the $4 \times 4$ versions of the matrices $A$ and $B$ from [24]. Using $V=0$ and

$$
A=\left[\begin{array}{cccc}
i / a & 0 & 0 & 0 \\
0 & i / a & 0 & i /(2 a) \\
0 & 0 & i /(2 a) & i /(2 a) \\
0 & 0 & i /(2 a) & i /(2 a)
\end{array}\right], \quad B=\left[\begin{array}{cccc}
0 & 0 & 0 & 0 \\
0 & 0 & 0 & 0 \\
0 & 0 & -1 / 2 & 1 / 2 \\
0 & 0 & 1 / 2 & -1 / 2
\end{array}\right],
$$

where $a$ is a real parameter, we obtain

$$
J(k)=\left[\begin{array}{cccc}
k / a & 0 & 0 & 0 \\
0 & k / a & 0 & k /(2 a) \\
0 & 0 & (k-a) /(2 a) & (k+a) /(2 a) \\
0 & 0 & (k+a) /(2 a) & (k-a) /(2 a)
\end{array}\right], \quad S(k)=S(0)=\left[\begin{array}{cccc}
1 & 0 & 0 & 0 \\
0 & 1 & 0 & 0 \\
0 & 0 & 0 & 1 \\
0 & 0 & 1 & 0
\end{array}\right] .
$$

On the other hand, we have $J(0)$ given by

$$
J(0)=\left[\begin{array}{cccc}
0 & 0 & 0 & 0 \\
0 & 0 & 0 & 0 \\
0 & 0 & -1 / 2 & 1 / 2 \\
0 & 0 & 1 / 2 & -1 / 2
\end{array}\right]
$$

The eigenvalues of $J(0)$ are $\lambda_{1}=0$ with $n_{1}=1, \lambda_{2}=0$ with $n_{2}=1, \lambda_{3}=0$ with $n_{3}=1$, and $\lambda_{4}=-1$ with $n_{4}=1$. We further have

$$
\begin{gathered}
\mu=\nu=3, \quad n=4, \quad P_{1}=P_{2}=I_{4}, \\
\mathcal{S}=\left[\begin{array}{cccc}
1 & 0 & 0 & 0 \\
0 & 1 & 0 & 0 \\
0 & 0 & 1 & -1 \\
0 & 0 & 1 & 1
\end{array}\right], \quad \mathcal{Z}(k)=\left[\begin{array}{cccc}
k / a & 0 & 0 & 0 \\
0 & k / a & k /(2 a) & k /(2 a) \\
0 & 0 & k / a & 0 \\
0 & 0 & 0 & -1
\end{array}\right],
\end{gathered}
$$




$$
\mathcal{A}_{1}=\left[\begin{array}{ccc}
1 / a & 0 & 0 \\
0 & 1 / a & 1 /(2 a) \\
0 & 0 & 1 / a
\end{array}\right], \quad \mathcal{B}_{1}=\left[\begin{array}{c}
0 \\
1 /(2 a) \\
0
\end{array}\right], \quad \mathcal{C}_{1}=\left[\begin{array}{lll}
0 & 0 & 0
\end{array}\right], \quad \mathcal{D}_{0}=[-1]
$$

Using the information in (7.9)-(7.11) in (6.23) we can verify that the right side in (6.23) coincides with $S(0)$ given in (7.8).

Example 7.4 The procedure in this example is again similar to that of Example 7.1. We use $V=0$, and for some real parameters $a$ and $b$, we let $A$ and $B$ be

$$
A=\left[\begin{array}{lll}
2 & 1 & a \\
0 & 0 & b \\
1 & 1 & c
\end{array}\right], \quad B=\left[\begin{array}{lll}
0 & 0 & 0 \\
0 & 0 & 1 \\
0 & 0 & 0
\end{array}\right]
$$

We obtain

$$
J(k)=\left[\begin{array}{ccc}
-2 i k & -i k & -i a k \\
0 & 0 & 1-i b k \\
-i k & -i k & -i c k
\end{array}\right], \quad S(k)=\left[\begin{array}{ccc}
1 & 0 & 0 \\
0 & \frac{-i+b k}{i+b k} & 0 \\
0 & 0 & 1
\end{array}\right]
$$

and hence

$$
S(0)=\left[\begin{array}{ccc}
1 & 0 & 0 \\
0 & -1 & 0 \\
0 & 0 & 1
\end{array}\right]
$$

On the other hand, the matrix $J(0)$, which given by

$$
J(0)=\left[\begin{array}{lll}
0 & 0 & 0 \\
0 & 0 & 1 \\
0 & 0 & 0
\end{array}\right],
$$

has eigenvalues $\lambda_{1}=0$ with $n_{1}=1$ and $\lambda_{2}=0$ with $n_{2}=2$. We further have

$$
\begin{gathered}
\mu=2, \quad \nu=3, \quad n=3, \quad P_{1}=I_{3}, \quad P_{2}=\left[\begin{array}{ccc}
1 & 0 & 0 \\
0 & 0 & 1 \\
0 & 1 & 0
\end{array}\right], \\
\mathcal{S}=\left[\begin{array}{lll}
1 & 0 & 0 \\
0 & 1 & 0 \\
0 & 0 & 1
\end{array}\right], \quad \mathcal{Z}(k)=\left[\begin{array}{ccc}
-2 i k & -i k & -i a k \\
-i k & -i k & -i c k \\
0 & 0 & 1-i b k
\end{array}\right],
\end{gathered}
$$




$$
\mathcal{A}_{1}=\left[\begin{array}{cc}
-2 i & -i \\
-i & -i
\end{array}\right], \quad \mathcal{B}_{1}=\left[\begin{array}{l}
-i a \\
-i c
\end{array}\right], \quad \mathcal{C}_{1}=\left[\begin{array}{ll}
0 & 0
\end{array}\right], \quad \mathcal{D}_{0}=[1]
$$

Using the information in (7.13)-(7.15) in (6.23) we can verify that the right hand side in (6.23) coincides with $S(0)$ given in (7.12).

Acknowledgment. The research leading to this article was supported in part by Consejo Nacional de Ciencia y Tecnología (CONACYT) under project CB2008-99100-F, the Texas Norman Hackerman Advanced Research Program (NHARP) under grant no. 003656-00462007, and by the Department of Defense under grant number DOD-BC063989.

\section{References}

[1] Z. S. Agranovich and V. A. Marchenko, The inverse problem of scattering theory, Gordon and Breach, New York, 1963.

[2] T. Aktosun, Factorization and small-energy asymptotics for the radial Schrödinger equation, J. Math. Phys. 41, 4262-4270 (2000).

[3] T. Aktosun and M. Klaus, Small-energy asymptotics for the Schrödinger equation on the line, Inverse Problems 17, 619-632 (2001).

[4] T. Aktosun, M. Klaus, and C. van der Mee, Small-energy asymptotics of the scattering matrix for the matrix Schrödinger equation on the line, J. Math. Phys. 42, 4627-4652 (2001).

[5] T. Aktosun and R. Weder, Inverse spectral-scattering problem with two sets of discrete spectra for the radial Schrödinger equation, Inverse Problems 22, 89-114 (2006).

[6] G. Berkolaiko, R. Carlson, S. A. Fulling, and P. Kuchment (eds.), Quantum graphs and their applications, Contemporary Mathematics, 415, Amer. Math. Soc., Providence, RI, 2006.

[7] J. Boman and P. Kurasov, Symmetries of quantum graphs and the inverse scattering problem, Adv. Appl. Math. 35, 58-70 (2005). 
[8] G. Borg, Uniqueness theorems in the spectral theory of $y^{\prime \prime}+(\lambda-q(x)) y=0$, Proc. 11th Scandinavian Congress of Mathematicians, Johan Grundt Tanums Forlag, Oslo, pp. 276-287, 1952.

[9] P. Deift and E. Trubowitz, Inverse scattering on the line, Commun. Pure Appl. Math. 32, 121-251 (1979).

[10] H. Dym, Linear algebra in action, Amer. Math. Soc., Providence, R.I., 2007.

[11] P. Exner, J. P. Keating, P. Kuchment, T. Sunada, and A. Teplyaev (eds.), Analysis on graphs and its applications, Proc. Symposia in Pure Mathematics, 77, Amer. Math. Soc., Providence, RI, 2008.

[12] L. D. Faddeev, Properties of the S-matrix of the one-dimensional Schrödinger equation, Amer. Math. Soc. Transl. 65 (ser. 2), 139-166 (1967).

[13] I. M. Gel'fand and B. M. Levitan, On the determination of a differential equation from its spectral function, Amer. Math. Soc. Transl. 1 (ser. 2), 253-304 (1955).

[14] N. I. Gerasimenko, The inverse scattering problem on a noncompact graph, Theoret. Math. Phys. 75, 460-470 (1988).

[15] N. I. Gerasimenko and B. S. Pavlov, A scattering problem on noncompact graphs, Theoret. Math. Phys. 74, 230-240 (1988).

[16] F. Gesztesy and B. Simon, Uniqueness theorems in inverse spectral theory for onedimensional Schrödinger operators, Transact. Amer. Math. Soc. 348, 349-373 (1996).

[17] B. Gutkin and U. Smilansky, Can one hear the shape of a graph? J. Phys. A 34, 6061-6068 (2001).

[18] M. S. Harmer, Inverse scattering for the matrix Schrödinger operator and Schrödinger operator on graphs with general self-adjoint boundary conditions, ANZIAM J. 44, 161168 (2002). 
[19] M. S. Harmer, The matrix Schrödinger operator and Schrödinger operator on graphs, Ph.D. thesis, University of Auckland, New Zealand, 2004.

[20] M. Harmer, Inverse scattering on matrices with boundary conditions, J. Phys. A 38, 4875-4885 (2005).

[21] M. Klaus, Low-energy behaviour of the scattering matrix for the Schrödinger equation on the line, Inverse Problems 4, 505-512 (1988).

[22] M. Klaus, Exact behavior of Jost functions at low energy, J. Math. Phys. 29, 148-154 (1988).

[23] V. Kostrykin and R. Schrader, Kirchhoff's rule for quantum wires, J. Phys. A 32, 595-630 (1999).

[24] V. Kostrykin and R. Schrader, Kirchhoff's rule for quantum wires. II: The inverse problem with possible applications to quantum computers, Fortschr. Phys. 48, 703-716 (2000).

[25] P. Kuchment, Quantum graphs. I. Some basic structures, Waves Random Media 14, S107-S128 (2004).

[26] P. Kuchment, Quantum graphs. II. Some spectral properties of quantum and combinatorial graphs, J. Phys. A 38, 4887-4900 (2005).

[27] P. Kurasov and M. Nowaczyk, Inverse spectral problem for quantum graphs, J. Phys. A 38, 4901-4915 (2005).

[28] P. Kurasov and F. Stenberg, On the inverse scattering problem on branching graphs, J. Phys. A 35, 101-121 (2002).

[29] B. M. Levitan, Inverse Sturm Liouville Problems, VNU Science Press, Utrecht, 1987.

[30] V. A. Marchenko, Some questions in the theory of one-dimensional linear differential operators of the second order, Amer. Math. Soc. Transl. 101 (ser. 2), 13-104 (1973).

[31] V. A. Marchenko, Sturm-Liouville operators and applications, Birkhäuser, Basel, 1986. 